\title{
REALIZATION THEORY FOR LINEAR AND BILINEAR SWITCHED SYSTEMS: A FORMAL POWER SERIES APPROACH PART II: BILINEAR SWITCHED SYSTEMS*
}

\author{
MihÁly PETRECZKY ${ }^{1}$
}

\begin{abstract}
This paper is the second part of a series of papers dealing with realization theory of switched systems. The current Part II addresses realization theory of bilinear switched systems. In Part I [Petreczky, ESAIM: COCV, DOI: 10.1051/cocv/2010014] we presented realization theory of linear switched systems. More precisely, in Part II we present necessary and sufficient conditions for a family of input-output maps to be realizable by a bilinear switched system, together with a characterization of minimal realizations. Similarly to Part I, the paper deals with two types of switched systems. The first one is when all switching sequences are allowed. The second one is when only a subset of switching sequences is admissible, but within this restricted set the switching times are arbitrary. The paper uses the theory of formal power series to derive the results on realization theory.
\end{abstract}

Mathematics Subject Classification. 93B15, 93B20, 93B25, 93C99.

Received July 29, 2005. Revised January 3rd, 2008 and September 12, 2009.

Published online March 31, 2010.

\section{INTRODUCTION}

The current paper is the second part of a series of papers addressing realization theory for linear and bilinear switched systems. Realization theory is one of central topics of systems theory. In addition to its theoretical relevance, realization theory has the potential of being applied for developing control, model reduction, and identification methods, as development of linear systems theory has demonstrated. Switched systems are a subclass of the so-called hybrid systems and they have been studied extensively, see [3].

In Part I [6] we presented realization theory for linear switched systems. In Part II we will present realization theory for bilinear switched systems. In both cases, we use an extension of the theory of rational formal power series. We refer to the introduction of Part I [6] for a more detailed introduction of the realization problem for switched system and the role of the theory of rational formal power series.

Contribution. As it was indicated in Part I, we present the following results on realization theory of bilinear switched systems.

Keywords and phrases. Hybrid systems switched linear systems, switched bilinear systems, realization theory, formal power series, minimal realization.

* The work presented in this paper is part of the author's Ph.D. thesis [5] and it was carried out during the author's stay at Centrum voor Wiskunde en Informatica (CWI), Amsterdam, The Netherlands.

1 Maastricht University, P.O. Box 616, 6200 MD Maastricht, The Netherlands. M.Petreczky@maastrichtuniversity.nl. 
- A bilinear switched system is a minimal realization of a set of input-output maps if and only if it is observable and semi-reachable from the set of states which induce the input-output maps. Minimal bilinear switched systems which realize a given set of input-output maps are unique up to similarity. Each bilinear switched system $\Sigma$ realizing a family of input-output maps $\Phi$ can be transformed to a minimal realization of $\Phi$.

- A set of input/output maps is realizable by a bilinear switched system if and only if it has a generalized Fliess-series expansion and the rank of its Hankel-matrix is finite. There is a procedure to construct a realization from the columns of the Hankel-matrix, and this procedure yields a minimal realization.

- Consider a set of input-output maps $\Phi$ defined on some subset of switching sequences. Assume that the switching sequences of this subset have arbitrary switching times and that their discrete mode parts form a regular language $L$. Then $\Phi$ has a realization by a bilinear switched system if and only if the $\Phi$ has a has a generalized Fliess-series expansion and its Hankel-matrix is of finite rank. There exists a procedure to construct an observable and semi-reachable realization from the columns of the Hankel-matrix. But this realization is not necessarily a realization with the smallest possible state-space dimension.

We will not discuss the algorithmic aspects of realization theory or partial realization theory in this paper. The main tool for deriving the results above is the theory of rational families of formal power series, explained in Part I [6]. The results of this paper were announced in [4], but [4] contains no detailed proofs.

Notation of the paper. Throughout the paper we will tacitly use the notation introduced in Part I [6]. In particular, we refer the reader to Part I for the definition of switched systems and formal power series.

Outline of the paper. In Section 2 we present the main results on bilinear switched systems. Section 3 presents the proof of the results on realization theory of bilinear switched systems. Finally, in Appendix A we present the proofs of certain technical results for bilinear switched systems.

\section{MAin RESUlts on REALIZATION THEORY FOR BILINEAR SWITCHED SYSTEMS}

The outline of the section is as follows. Section 2.1 recalls the basic results from realization theory of bilinear systems. These results will be used later on for realization theory of bilinear switched systems. In Section 2.2 we present the definition and some basic properties observability and reachability properties of bilinear switched systems. Section 2.3 presents the main results on minimality of bilinear switched systems, both for arbitrary and constrained switching. Section 2.4 states the necessary and sufficient conditions for existence of a realization by a bilinear switched system.

\subsection{Review of realization theory of bilinear systems}

Below we recall from $[1,2]$ some basic results on realization theory of bilinear systems. A bilinear control system is a continuous-time control system determined by the following equations

$$
\dot{x}(t)=A x(t)+\sum_{j=1}^{m}\left(B_{j} x(t)\right) u_{j}(t), x(0)=x_{0} \text { and } y(t)=C x(t) .
$$

Here the state-space is the space $\mathcal{X}=\mathbb{R}^{n}, n>0$, the input space is $\mathcal{U}=\mathrm{R}^{m}$, and the output space is $\mathcal{Y}=\mathbb{R}^{p}$. $x(t) \in \mathcal{X}$ is the state at time $t \in T, y(t) \in \mathcal{Y}$ is the output at time $t \in T$. The input $u(t) \in \mathcal{U}$ at time $t \in T$ is assumed to be of the form $u(t)=\left(u_{1}(t), u_{2}(t), \ldots, u_{m}(t)\right)^{T}$, i.e. $u_{i}(t)$ is just the $i$ th coordinate of the vector $u(t)$. In addition, it is assumed that the input function $u: T \ni t \mapsto u(t) \in \mathcal{U}$ is piecewise-continuous, i.e. $u \in P C(T, \mathcal{U})$. Furthermore, $A, B_{j}, j=1, \ldots, m$ and $C$ are matrices $A \in \mathbb{R}^{n \times n}, B_{j} \in \mathbb{R}^{n \times n}$, and $C \in \mathbb{R}^{p \times n}$.

Let $x(0)=x_{0} \in \mathcal{X}$ be the initial state of the system (2.1). Denote by $x\left(x_{0}, u, t\right)$ the state at time $t$ if the system (2.1) was started at time 0 with the input map $u \in P C(T, \mathcal{U})$. Denote the corresponding output by $y\left(x_{0}, u, t\right)$, i.e. $y\left(x_{0}, u, t\right)=C x\left(x_{0}, u, t\right)$. We say that a map $f: P C(T, \mathcal{U}) \times T \rightarrow \mathcal{Y}$ is realized by the bilinear 
system (2.1), if $f(u, t)=y\left(x_{0}, u, t\right)$ for any $u \in P C(T, \mathcal{U})$ and $t \in T$. The bilinear system (2.1) is called a minimal realization of $f$, if its state-space dimension is the smallest possible among all the bilinear systems which realize $f$.

Below we recall from $[1,2]$ the representation of state and output trajectories of bilinear systems as infinite series of iterated integrals.

Notation 2.1 (iterated integral). For each input vector $u=\left(u_{1}, u_{2}, \ldots, u_{m}\right)^{T} \in \mathcal{U}=\mathbb{R}^{m}$ denote by $\mathrm{d} \zeta_{j}[u]$, $j=0,1,2, \ldots, m$ the following scalars $\mathrm{d} \zeta_{j}[u]=u_{j} \in \mathbb{R}, j=1,2, \ldots, m, \mathrm{~d} \zeta_{0}[u]=1$. Let $\mathrm{Z}_{m}=\{0,1, \ldots, m\}$ and in accordance with [6], Section 2.1, denote by $\mathrm{Z}_{m}^{*}$ the set of all finite words over $\mathrm{Z}_{m}$. For each word $w \in \mathrm{Z}_{m}^{*}$, time instance $t \in T$, and input $u \in P C(T, \mathcal{U})$ define the iterated integral $V_{w}[u](t) \in \mathbb{R}$ recursively as follows:

$$
V_{w}[u](t)= \begin{cases}1 & \text { if } w=\epsilon \\ \int_{0}^{t} \mathrm{~d} \zeta_{j}[u(\tau)] V_{s}[u](\tau) \mathrm{d} \tau & \text { if } w=s j \text { for some word } s \in \mathrm{Z}_{m}^{*} \text { and integer } j \in \mathrm{Z}_{m} .\end{cases}
$$

Notation 2.2. Denote the matrix $A$ of the bilinear system (2.1) by $B_{0}$.

With the notation above, the output and state trajectory of a bilinear system are of the form

$$
\begin{aligned}
& x\left(x_{0}, u, t\right)=x_{0}+\sum_{k=1}^{\infty} \sum_{i_{1}, i_{2}, \ldots, i_{k}=0}^{m} B_{i_{k}} B_{i_{k-1}} \ldots B_{i_{1}} x(0) V_{i_{1} i_{2} \ldots i_{k}}[u](t) \\
& y\left(x_{0}, u, t\right)=C x_{0}+\sum_{k=1}^{\infty} \sum_{i_{1}, i_{2}, \ldots, i_{k}=0}^{m} C B_{i_{k}} B_{i_{k-1}} \ldots B_{i_{1}} x(0) V_{i_{1} i_{2} \ldots i_{k}}[u](t) .
\end{aligned}
$$

The bilinear system (2.1) is span-reachable, if the linear span of all the states which are reachable from the initial state $x_{0}$ yields the whole state-space. A bilinear system is observable if there are no indistinguishable states, i.e. there exist no states $x_{1} \neq x_{2}$ such that $y\left(x_{1}, u, t\right)=y\left(x_{2}, u, t\right)$ for all $u \in P C(T, \mathcal{U}), t \in T$. A bilinear system of the form (2.1) is span-reachable if and only if

$$
n=\operatorname{dim} \operatorname{Span}\left(\left\{B_{i_{k}} B_{i_{k-1}} \ldots B_{i_{1}} x_{0} \mid i_{1}, i_{2}, \ldots, i_{k}=0, \ldots, m, k>0\right\} \cup\left\{x_{0}\right\}\right) .
$$

A bilinear system (2.1) is observable (has no pair of indistinguishable states) if and only if

$$
\operatorname{ker} C \cap \bigcap_{k=1}^{\infty} \bigcap_{i_{1}, \ldots, i_{k}=0}^{m} \operatorname{ker} C B_{i_{k}} B_{i_{k-1}} \ldots B_{i_{1}}=\{0\}
$$

Both span-reachability and observability of bilinear systems can be checked numerically [1,2]. Minimality of bilinear systems can fully be characterized as follows; a bilinear system is minimal if and only if it is spanreachable and observable. Moreover, minimal bilinear realizations of the same input-output map are isomorphic.

As the next step, we recall the necessary and sufficient conditions for existence of a realization by a bilinear system. To this end, let us recall the notation of classical Fliess-series expansion from [1,2] $u$ from (2.2). We denote by $\mathrm{Z}_{m}^{*}$ the set of all finite sequences of elements of $\mathrm{Z}_{m}$. We call a map $c: \mathrm{Z}_{m}^{*} \rightarrow \mathcal{Y}$ convergent generating series if there exists reals $K, M>0$ such that for all $s \in \mathrm{Z}_{m}^{*},\|c(s)\|_{2} \leq K M^{|s| 2}$. It can be shown that the series $F_{c}(u, t)=\sum_{k=0}^{\infty} \sum_{j_{1}, j_{2}, \ldots j_{k}=0}^{m} c\left(j_{1} j_{2} \ldots j_{k}\right) V_{j_{1} j_{2} \ldots j_{k}}[u](t)$, where $u \in \bar{P} C(T, \mathcal{U})$ is absolutely convergent and it uniquely defines a map $F_{c}: P C(T, \mathcal{U}) \times T \ni(u, t) \mapsto F_{c}(u, t) \in \mathcal{Y}$. Moreover if $F_{c}=F_{d}$ then $c=d$. We say that an input-output map $f: P C(T, \mathcal{U}) \times T \rightarrow \mathbb{R}^{p}$ admits a Fliess-series expansion, if there exists a generating convergent series $c_{f}: \mathrm{Z}_{m}^{*} \rightarrow \mathcal{Y}$ such that $f=F_{c_{f}}$. We refer to the series $c_{f}$ as the generating series of $f$.

\footnotetext{
${ }^{2}$ In the literature, usually the weaker condition $\|c(s)\|_{2} \leq|s| ! K M^{|s|}$ is required instead. However, for our purposes it is more convenient to require the stronger condition $\|c(s)\|_{2} \leq K M^{|s|}$.
} 
It can be shown $f$ is realized by a bilinear systems (2.1) if and only if $f$ has a Fliess-series expansion and the generating series $c_{f}$ of $f$ is of the form

$$
c_{f}(\epsilon)=C x_{0} \text { and } c_{f}\left(j_{1} j_{2} \ldots j_{k}\right)=C B_{j_{k}} B_{j_{k-1}} \ldots B_{j_{1}} x_{0} \text { for all } j_{1}, j_{2}, \ldots, j_{k} \in \mathrm{Z}_{m}, k>0 .
$$

That is, existence of a bilinear realization of $f$ is equivalent to the existence of matrices $B_{j}, C, j \in \mathrm{Z}_{m}$ and a vector $x_{0}$ such that (2.6) holds. The latter problem is related to the problem of existence of a rational representation of a formal power series. We define the Hankel-matrix $H_{f}$ of $f$ as the infinite block matrix, columns and rows of which are indexed by sequences $v \in \mathrm{Z}_{m}^{*}$. The $p \times 1$ block entry of $H_{f}$ lying on the intersection of the row indexed by $v$ and the column indexed by $w$ equals $c(w v)$. It turns out that the generating series $c$ has a representation of the form (2.6) if and only if the column rank of $H_{f}$ is finite. That is, $f$ has a realization by a bilinear system if and only if $f$ has a Fliess-series expansion and the column rank of its Hankel-matrix $H_{f}$ is finite. Realization theory of bilinear systems can be developed by using the theory of rational formal power series, see $[1,2,7]$.

\subsection{Definition and basic properties of bilinear switched systems}

Below we present the definition and elementary properties of bilinear switched systems.

Definition 2.1 (bilinear switched systems). A bilinear switched system $\Sigma$ is a switched system of the form [6], equation (2.1) such that for each discrete mode $q \in Q$ and input $u=\left(u_{1}, \ldots, u_{m}\right)^{T} \in \mathcal{U}$,

$$
f_{q}(x, u)=A_{q} x+\sum_{j=1}^{m}\left(B_{q, j} x\right) u_{j} \text { and } h_{q}(x)=C_{q} x
$$

where $C_{q}, A_{q}, B_{q, j}, j=1, \ldots, m$ are matrices of the form $A_{q} \in \mathbb{R}^{n \times n}, B_{q, j} \in \mathbb{R}^{n \times n}, j=1, \ldots, m$ and $C_{q} \in \mathbb{R}^{p \times n}$. We denote the bilinear switched system above by $\Sigma=\left(\mathcal{X}, \mathcal{U}, \mathcal{Y}, Q,\left\{\left(A_{q},\left\{B_{q, j}\right\}_{j=1,2, \ldots, m}, C_{q}\right) \mid q \in Q\right\}\right)$. Recall that $\mathcal{X}=\mathbb{R}^{n}, n>0$ is the state-space, $Q$ is the finite set of discrete modes, $\mathcal{U}=\mathbb{R}^{m}, m>0$ is the set of continuous inputs and $\mathcal{Y}=\mathbb{R}^{p}, p>0$ is the set of continuous outputs of $\Sigma$.

Notation 2.3. In the rest of the paper we use the symbols $\mathcal{U}=\mathbb{R}^{m}, \mathcal{Y}=\mathbb{R}^{p}$ and $Q$ to denote the continuousvalued inputs, outputs and the set of discrete modes respectively.

That is, a bilinear switched system is a switched system, continuous subsystems of which are bilinear control systems. Since we agreed to view bilinear switched systems as switched systems of the form [6], equation (2.1), the same definition of the semantics (evolution of the state and output) apply as the one defined in [6], Section 2, for switched systems. In particular, we view the switching signal as part of the input rather than part of the state. Furthermore, the definition of semi-reachability, observability, input-output map, and realization presented in [6], Section 2, apply as well. Next, we define the concept of minimality for bilinear switched system. To this end, recall from [6], Section 2, that the dimension of a bilinear switched system equals the dimension of its state-space. Let $\Phi$ be a family of input-output maps. A bilinear switched system realization is a switched system realization $(\Sigma, \mu)$ such that $\Sigma$ is a bilinear switched system.

Definition 2.2 (minimality). A bilinear switched system realization $(\Sigma, \mu)$ is a minimal realization of $\Phi$, if $(\Sigma, \mu)$ is a realization of $\Phi$ and for any bilinear switched system realization $(\hat{\Sigma}, \hat{\mu})$ of $\Phi, \operatorname{dim} \Sigma \leq \operatorname{dim} \hat{\Sigma}$. A bilinear switched system $\Sigma$ is a minimal realization of $\Phi$, if $(\Sigma, \mu)$ is a minimal realization of $\Phi$ for some $\mu$.

In simple words, a bilinear switched system is a minimal realization of $\Phi$ if it has the smallest dimensional state-space among all the bilinear switched systems which are realizations of $\Phi$. A bilinear switched system can be a minimal realization for $\Phi$ and can fail to be a minimal realization for another family of input-output maps. As the next step, we will introduce the notion of bilinear switched system morphism. 
Definition 2.3 (bilinear switched systems morphisms). Let $\Sigma_{1}=\left(\mathcal{X}_{1}, \mathcal{U}, \mathcal{Y}, Q,\left\{\left(A_{q}^{1},\left\{B_{q, j}^{1}\right\}_{j=1,2, \ldots, m}, C_{q}^{1}\right) \mid q \in\right.\right.$ $Q\})$ and $\Sigma_{2}=\left(\mathcal{X}_{2}, \mathcal{U}, \mathcal{Y}, Q,\left\{\left(A_{q}^{2},\left\{B_{q, j}^{2}\right\}_{j=1,2, \ldots, m}, C_{q}^{2}\right) \mid q \in Q\right\}\right)$ be two bilinear switched systems. Let $\Phi$ be a family of input-output maps (possibly with constrained switching) and consider maps $\mu_{i}: \Phi \rightarrow \mathcal{X}_{i}, i=1,2$. A linear map $T: \mathcal{X}_{1} \rightarrow \mathcal{X}_{2}$ is called a bilinear switched system morphism from $\left(\Sigma_{1}, \mu_{1}\right)$ to $\left(\Sigma_{2}, \mu_{2}\right)$, denoted by $T:\left(\Sigma_{1}, \mu_{1}\right) \rightarrow\left(\Sigma_{2}, \mu_{2}\right)$, if it commutes with the matrices of $\Sigma_{1}$ and $\Sigma_{2}$ and with the maps $\mu_{1}$ and $\mu_{2}$, i.e.

$$
T A_{q}^{1}=A_{q}^{2} T \quad C_{q}^{1}=C_{q}^{2} T \quad T B_{q, j}^{1}=B_{q, j}^{2} T \quad T \mu_{1}(f)=\mu_{2}(f) \quad \text { for all } q \in Q, j=1,2, \ldots, m, f \in \Phi .
$$

$T$ is said to be injective, surjective, an isomorphism, if it is injective, surjective, resp. an isomorphism if viewed as a linear map $T: \mathcal{X}_{1} \rightarrow \mathcal{X}_{2}$. If there exists an isomorphism $T:\left(\Sigma_{1}, \mu_{1}\right) \rightarrow\left(\Sigma_{2}, \mu_{2}\right)$, then $\left(\Sigma_{1}, \mu_{1}\right)$ and $\left(\Sigma_{2}, \mu_{2}\right)$ are said to be isomorphic.

Next, we present the representation of state and output trajectories of a bilinear switched system in terms of infinite sum of iterated integrals. To this end, we need additional notation.

Notation 2.4 (product of iterated integrals). For each words $w_{1}, w_{2}, \ldots, w_{k} \in \mathrm{Z}_{m}^{*}, k>0$, time instances $\left(t_{1}, t_{2}, \ldots, t_{k}\right) \in T^{k}$, and for input $u \in P C(T, \mathcal{U})$ define $V_{w_{1}, w_{2}, \ldots, w_{k}}[u]\left(t_{1}, t_{2}, \ldots, t_{k}\right) \in \mathbb{R}$ by

$$
V_{w_{1}, \ldots, w_{k}}[u]\left(t_{1}, t_{2}, \ldots, t_{k}\right)=V_{w_{1}}[u]\left(t_{1}\right) V_{w_{2}}\left[\operatorname{Shift}_{1}(u)\right]\left(t_{2}\right) \ldots V_{w_{k}}\left[\operatorname{Shift}_{k-1}(u)\right]\left(t_{k}\right)
$$

where $\operatorname{Shift}_{i}(u)(s)=u\left(s+\sum_{j=1}^{i} t_{j}\right)$ for all $s \in T$ and $i=1, \ldots, k-1$.

Informally, $V_{w_{1}, \ldots, w_{k}}[u]\left(t_{1}, \ldots, t_{k}\right)$ is just the product of the iterated integrals $V_{w_{1}}[u]\left(t_{1}\right), V_{w_{2}}\left[\operatorname{Shift}_{1}(u)\right]\left(t_{2}\right)$, etc. of $u$. The iterated integral $V_{w_{1}}[u]\left(t_{1}\right)$ is supposed to capture the evolution of the system at some discrete mode $q_{1}$, the iterated integral $V_{w_{2}}\left[\operatorname{Shift}_{1}(u)\right]\left(t_{2}\right)$ is supposed to capture the evolution of the system at the subsequent discrete mode $q_{2}$ and so on. The times $t_{1}, \ldots, t_{k}$ reflect the time spent in discrete modes $q_{1}, q_{2}, \ldots, q_{k}$.

Notation 2.5 (products of system matrices). Let $I_{n}$ be the $n \times n$ identity matrix. For each $q \in Q$ and for any sequence $w \in \mathrm{Z}_{m}^{*}$, define the matrix $B_{q, w}$ as follows. If $w=\epsilon$, then $B_{q, w}=B_{q, \epsilon}=I_{n}$, and if $w=j_{1} j_{2} \ldots j_{k}$, for some $k>0$, and $j_{1}, \ldots, j_{k} \in \mathrm{Z}_{m}$, then

$$
B_{q, w}=B_{q, j_{k}} B_{q, j_{k-1}} \ldots B_{q, j_{1}} \text { where } B_{q, 0}=A_{q} .
$$

The precise form of iterated integral representation of the state and output trajectories is as follows.

Theorem 2.1 (state and output trajectories). For each state $x_{0} \in \mathcal{X}$, input $u \in P C(T, \mathcal{U})$ and switching sequence $s=\left(q_{1}, t_{1}\right)\left(q_{2}, t_{2}\right) \ldots\left(q_{k}, t_{k}\right) \in(Q \times T)^{+}, q_{1}, q_{2}, \ldots, q_{k} \in Q, t_{1}, t_{2}, \ldots, t_{k} \in T, k>0$

$$
\begin{aligned}
& x_{\Sigma}\left(x_{0}, u, s\right)=\sum_{w_{1}, w_{2}, \ldots, w_{k} \in \mathrm{Z}_{m}^{*}}\left(B_{q_{k}, w_{k}} B_{q_{k-1}, w_{k-1}} \ldots B_{q_{1}, w_{1}} x_{0}\right) V_{w_{1}, w_{2}, \ldots, w_{k}}[u]\left(t_{1}, t_{2}, \ldots, t_{k}\right) \\
& y_{\Sigma}\left(x_{0}, u, s\right)=\sum_{w_{1}, w_{2}, \ldots, w_{k} \in \mathrm{Z}_{m}^{*}}\left(C_{q_{k}} B_{q_{k}, w_{k}} B_{q_{k-1}, w_{k-1}} \ldots B_{q_{1}, w_{1}} x_{0}\right) V_{w_{1}, w_{2}, \ldots, w_{k}}[u]\left(t_{1}, t_{2}, \ldots, t_{k}\right) .
\end{aligned}
$$

The proof of the theorem can be found in Appendix A.2. We conclude the section with a numerical example.

Example 2.1. Let $\Sigma$ be a bilinear switched system of the form (2.7) where $Q=\left\{\sigma_{1}, \sigma_{2}\right\}$, the state-space is $\mathcal{X}=\mathbb{R}^{3}, \mathcal{U}=\mathbb{R}^{2}, \mathcal{Y}=\mathbb{R}$ and the system matrices are as follows:

$$
\begin{aligned}
& A_{\sigma_{1}}=\left[\begin{array}{lll}
1 & 0 & 0 \\
0 & 2 & 0 \\
0 & 0 & 2
\end{array}\right], B_{\sigma_{1}, 1}=\left[\begin{array}{lll}
1 & 0 & 0 \\
0 & 5 & 0 \\
0 & 0 & 1
\end{array}\right], B_{\sigma_{1}, 2}=\left[\begin{array}{lll}
1 & 0 & 0 \\
0 & 3 & 1 \\
0 & 0 & 1
\end{array}\right], C_{\sigma_{1}}=\left[\begin{array}{lll}
1 & 1 & 0
\end{array}\right] \\
& A_{\sigma_{2}}=\left[\begin{array}{lll}
2 & 0 & 0 \\
0 & 1 & 0 \\
0 & 0 & 1
\end{array}\right], B_{\sigma_{2}, 1}=\left[\begin{array}{lll}
5 & 0 & 0 \\
0 & 1 & 0 \\
0 & 0 & 1
\end{array}\right], B_{\sigma_{2}, 2}=\left[\begin{array}{lll}
3 & 0 & 1 \\
0 & 0 & 1 \\
0 & 0 & 0
\end{array}\right], C_{\sigma_{2}}=\left[\begin{array}{lll}
1 & 1 & 0
\end{array}\right] .
\end{aligned}
$$


Consider the states $x_{0,1}=(1,0,0)^{T} \in \mathbb{R}^{3}$ and $x_{0,2}=(0,1,0)^{T} \in \mathbb{R}^{3}$. Consider the input-output maps $f_{i}=$ $y_{\Sigma}\left(x_{0, i},.\right), i=1,2$ induced by the initial state $x_{0, i}, i=1,2$. Let $\Phi=\left\{f_{1}, f_{2}\right\}$ and define the map $\mu: \Phi \rightarrow \mathbb{R}^{3}$ as $\mu\left(f_{i}\right)=x_{0, i}, i=1,2$. It is immediate from the definition that $(\Sigma, \mu)$ realizes $\Phi$. The state and output trajectories induces by $x_{0, i}, i=1,2$ admit the following representation. For input $u \in P C(T, \mathcal{U})$, switching sequence $w=\left(q_{1}, t_{1}\right)\left(q_{2}, t_{2}\right) \ldots\left(q_{k}, t_{k}\right) \in(Q \times T)^{+}$,

$$
\begin{aligned}
& x_{\Sigma}\left(x_{0, i}, u, w\right)=\sum_{w_{1}, w_{2}, \ldots, w_{k} \in Z_{m}^{*}} d_{x_{0, i}}\left(\left(q_{1}, w_{1}\right)\left(q_{2}, w_{2}\right) \ldots\left(q_{k}, w_{k}\right)\right) V_{w_{1}, \ldots, w_{k}}[u]\left(t_{1}, \ldots, t_{k}\right) \\
& y_{\Sigma}\left(x_{0, i}, u, w\right)=\sum_{w_{1}, w_{2}, \ldots, w_{k} \in Z_{m}^{*}} c_{x_{0, i}}\left(\left(q_{1}, w_{1}\right)\left(q_{2}, w_{2}\right) \ldots\left(q_{k}, w_{k}\right)\right) V_{w_{1}, \ldots, w_{k}}[u]\left(t_{1}, \ldots, t_{k}\right)
\end{aligned}
$$

where for each $s=\left(q_{1}, w_{1}\right) \ldots\left(q_{k}, w_{k}\right), d_{x_{0, i}}(s) \in \mathbb{R}^{3}$ and $c_{x_{0, i}}(s) \in \mathbb{R}$ are as follows. For any $(q, j) \in Q \times \mathrm{Z}_{m}$ denote by $\#_{(q, j)} s$ the sum of number of occurrences of $j$ in $w_{i}$ for all $i=1, \ldots, k$ such that $q_{i}=q$ :

$$
\begin{aligned}
& d_{x_{0,1}}(s)=3^{\#\left(\sigma_{2}, 1\right) s} 5^{\#\left(\sigma_{2}, 2\right)} s 2^{\#\left(\sigma_{2}, 0\right)} s(1,0,0)^{T} \text { and } d_{x_{0,2}}(s)=3^{\#\left(\sigma_{1}, 1\right) s} 5^{\#\left(\sigma_{1}, 2\right)} s 2^{\#\left(\sigma_{1}, 0\right)} s(0,1,0)^{T} \\
& c_{x_{0,2}}(s)=3^{\#\left(\sigma_{2}, 1\right)} s 5^{\#\left(\sigma_{2}, 2\right)} s 2^{\#\left(\sigma_{2}, 0\right)} s \text { and } c_{x_{0,2}}(s)=3^{\#\left(\sigma_{1}, 1\right)} s 5^{\#\left(\sigma_{1}, 2\right)} s 2^{\#\left(\sigma_{1}, 0\right)} s \text {. }
\end{aligned}
$$

In the sequel, we will come back to $(\Sigma, \mu)$.

\subsection{Minimality, observability and semi-reachability}

\subsubsection{Conditions for observability and semi-reachability}

Semi-reachability and observability of bilinear switched systems can be characterized as follows.

Theorem 2.2 (semi-reachability and observability). Let $\Sigma$ be a bilinear switched system of the form (2.7) and let $\mathcal{X}_{0} \subseteq \mathcal{X}$ a subset of initial states.

(i) Let $W\left(\mathcal{X}_{0}\right)=\operatorname{Span}\left\{x \in \mathcal{X} \mid x \in \operatorname{Reach}\left(\mathcal{X}_{0}, \Sigma\right)\right\}$ be the linear space spanned by the states reachable from $\mathcal{X}_{0}$. Then,

$$
W\left(\mathcal{X}_{0}\right)=\operatorname{Span}\left\{B_{q_{k}, w_{k}} B_{q_{k-1}, w_{k-1}} \ldots B_{q_{1}, w_{1}} x_{0} \mid q_{1}, \ldots, q_{k} \in Q, k>0, w_{1}, \ldots, w_{k} \in \mathrm{Z}_{m}^{*}, x_{0} \in \mathcal{X}_{0}\right\}
$$

The system $\Sigma$ is semi-reachable from $\mathcal{X}_{0}$ if and only if $\operatorname{dim} W\left(\mathcal{X}_{0}\right)=\operatorname{dim} \mathcal{X}$.

(ii) Define the observability kernel $O_{\Sigma}$ of $\Sigma$ as the intersection of kernels of $C_{q_{k}} B_{q_{k}, w_{k}} \ldots B_{q_{1}, w_{1}}$, i.e.

$$
O_{\Sigma}=\bigcap_{q_{1}, \ldots, q_{k} \in Q, k>0, w_{1}, \ldots, w_{k} \in \mathrm{Z}_{m}^{*}} \operatorname{ker} C_{q_{k}} B_{q_{k}, w_{k}} \ldots B_{q_{1}, w_{1}}
$$

Any two states $x_{1}, x_{2} \in \mathcal{X}$ of $\Sigma$ are indistinguishable if and only if $x_{1}-x_{2} \in O_{\Sigma}$. The system $\Sigma$ is observable if and only if $O_{\Sigma}=\{0\}$.

The proof of Theorem 2.2 is presented in Appendix A. Informally, Theorem 2.2 states that (i) the linear space spanned by the states reachable from $\mathcal{X}_{0}$ equals the vector space spanned by all the matrix products of the form $B_{q_{k}, w_{k}} B_{q_{k-1}, w_{k-1}} \ldots B_{q_{1}, w_{1}} x_{0}, x_{0} \in \mathcal{X}_{0}$, and (ii) two states $x_{1}$ and $x_{2}$ are indistinguishable, if $x_{1}-x_{2}$ belong to the intersection of the kernels of all the matrices $C_{q_{k}} B_{q_{k}, w_{k}} B_{q_{k-1}, w_{k-1}} \ldots B_{q_{1}, w_{1}}$. By means of a counterexample, it is easy to see that observability or semi-reachability of a bilinear switched system does not imply observability or span-reachability of any of its bilinear subsystems.

Remark 2.1 (algorithms). Semi-reachability and observability presented above can be checked numerically. There is also a numerical algorithm for transforming any bilinear switched system realization to a semi-reachable and observable one. See [5] for further details.

\subsubsection{Minimality for arbitrary switching}

Consider a family of input-output maps $\Phi$ with arbitrary switching. 
Theorem 2.3 (minimality: arbitrary switching). Assume that $\left(\Sigma_{\min }, \mu_{\min }\right)$ is a bilinear switched system realization of $\Phi$. Then the following are equivalent:

(i) $\left(\Sigma_{\min }, \mu_{\min }\right)$ is a minimal bilinear switched system realization of $\Phi$;

(ii) $\left(\Sigma_{\min }, \mu_{\min }\right)$ is semi-reachable and it is observable;

(iii) The dimension of $\Sigma_{\text {min }}$ equals the rank of the Hankel-matrix $H_{\Phi}$ of $\Phi$, i.e. $\operatorname{dim} \Sigma_{\min }=\operatorname{rank} H_{\Phi}$. Here, the Hankel-matrix of $\Phi$ and its rank will be defined in Definition 2.8 later on;

(iv) For any semi-reachable bilinear switched system realization $(\Sigma, \mu)$ of $\Phi$, there exist a surjective bilinear switched system morphism $T:(\Sigma, \mu) \rightarrow\left(\Sigma_{\min }, \mu_{\min }\right)$.

All minimal bilinear switched system realizations of $\Phi$ are isomorphic.

The proof of Theorem 2.3 will be presented in Section 3.1. Note that minimality of a bilinear switched system does not imply minimality of any of its bilinear subsystems.

Remark 2.2 (minimization algorithms). From Remark 2.1, it follows that minimality can be checked by a numerical algorithm. In addition, any bilinear switched system can be transformed to a minimal one realizing the same input-output maps, see Section 3.1. Moreover, this transformation can be made effective, see [5].

Example 2.2. Recall from Example 2.1 the definition of the realization $(\Sigma, \mu)$ and the set of input-output maps $\Phi=\left\{f_{1}, f_{2}\right\}$. Below we will present the result $\left(\Sigma_{m}, \mu_{m}\right)$ of the minimization procedure applied to $(\Sigma, \mu)$. Assume that $\Sigma_{m}=\left\{\left(\mathcal{X}_{m}, \mathcal{U}, \mathcal{Y}, Q,\left\{\left(A_{q}^{m},\left\{B_{q, j}^{m}\right\}_{j=1,2, \ldots, m}, C_{q}^{m}\right) \mid q \in Q\right\}\right)\right.$. The set of discrete modes $Q=\left\{q_{1}, q_{2}\right\}$ of $\Sigma_{m}$ is the same as in $\Sigma$, the state-space of $\Sigma_{m}$ is $\mathcal{X}_{m}=\mathbb{R}^{2}$ and the matrices of $\Sigma_{m}$ are of the following form:

$$
\begin{aligned}
& A_{\sigma_{1}}^{m}=\left[\begin{array}{cc}
1 & -0 \\
0 & 2
\end{array}\right], B_{\sigma_{1}, 1}^{m}=\left[\begin{array}{ll}
1 & 0 \\
0 & 5
\end{array}\right], B_{\sigma_{1}, 2}^{m}=\left[\begin{array}{ll}
1 & 0 \\
0 & 3
\end{array}\right], C_{\sigma_{1}}^{m}=\left[\begin{array}{c}
1 \\
-1
\end{array}\right]^{T}, \\
& A_{\sigma_{2}}^{m}=\left[\begin{array}{ll}
2 & 0 \\
0 & 1
\end{array}\right], B_{\sigma_{2}, 1}^{m}=\left[\begin{array}{ll}
5 & 0 \\
0 & 1
\end{array}\right], B_{\sigma_{2}, 2}^{m}=\left[\begin{array}{ll}
3 & 0 \\
0 & 1
\end{array}\right], C_{\sigma_{2}}^{m}=\left[\begin{array}{c}
1 \\
-1
\end{array}\right]^{T} .
\end{aligned}
$$

Define the map $\mu_{m}: \Phi \rightarrow \mathbb{R}^{2}$ as $\mu_{m}\left(f_{1}\right)=(1,0)^{T}$ and $\mu_{m}\left(f_{2}\right)=(0,-1)^{T}$.

\subsubsection{Minimality for constrained switching}

Let $L \subseteq Q^{+}$be a set of admissible sequences of discrete modes, and let $\Phi \subseteq F(P C(T, \mathcal{U}) \times T L, \mathcal{Y})$ be a family of input-output maps with the switching constraint $L$. Recall the definition of the $\operatorname{set} \operatorname{comp}(L) \operatorname{from}[6]$, equation (3.7),

$$
\operatorname{comp}(L)=\left\{q_{1} q_{2} \ldots q_{k} \in Q^{+} \mid q_{1}, q_{2}, \ldots, q_{k} \in Q, k \geq 1, \forall v \in Q^{*}: v q_{k} \notin L\right\} .
$$

The set $\operatorname{comp}(L)$ contains those sequences of discrete modes, for which no information on the behavior of the switched system can be obtained by observing the behavior of the system for admissible switching sequences. If we apply [6], Definition 2.11, to $\operatorname{comp}(L)$ instead of $L$, we obtain the set $T(\operatorname{comp}(L))$ of all the switching sequences for which the sequence of discrete modes belong to $\operatorname{comp}(L)$, i.e. $T(\operatorname{comp}(L))=\left\{\left(q_{1}, t_{1}\right) \ldots\left(q_{k}, t_{k}\right) \in\right.$ $\left.(Q \times T)^{+} \mid q_{1}, \ldots, q_{k} \in Q, t_{1}, \ldots, t_{k} \in T, q_{1} q_{2} \ldots q_{k} \in \operatorname{comp}(L), k \geq 1\right\}$.

Theorem 2.4 (minimality: constrained switching). Assume that $L$ is a regular language. If $\Phi$ has a realization by a bilinear switched system, then there exists a bilinear switched system realization $\left(\Sigma_{m}, \mu_{m}\right)$ of $\Phi$, such that $\left(\Sigma_{m}, \mu_{m}\right)$ is observable and semi-reachable and

$$
\forall f \in \Phi, u \in P C(T, \mathcal{U}), w \in T(\operatorname{comp}(L)): y_{\Sigma_{m}}\left(\mu_{m}(f), u, w\right)=0 .
$$

In addition, there exists a constant $M>0$ such that for any bilinear switched system realization $(\Sigma, \mu)$ of $\Phi$

$$
\operatorname{dim} \Sigma_{m} \leq M \cdot \operatorname{dim} \Sigma \text {. }
$$

The constant $M$ depends only on the set $L$. 
The proof of Theorem 2.4 will be presented in Section 3.2. A bilinear switched system realization $(\Sigma, \mu)$ of $\Phi$ is called quasi-minimal, if $(\Sigma, \mu)$ is semi-reachable and observable and it satisfies $(2.16)$ and (2.17).

Remark 2.3 (algorithms). Any bilinear switched system realization of $\Phi$ can be converted to a quasi-minimal realization of $\Phi$. This transformation will be elaborated on in Section 3.2, and it can be formulated as a numerical algorithm, see [5].

Remark 2.4 (quasi-minimality is not sufficient for minimality). It is possible to show by means of a counterexample that quasi-minimality is not sufficient for minimality. In Example 2.3 a set $L$, and family of input-output maps $\Phi \subseteq F(P C(T, \mathcal{U}) \times T L, \mathcal{Y})$ is presented, such that $\left(\Sigma_{m}, \mu_{m}\right)$ and $\left(\Sigma_{c}, \mu_{c}\right)$ are both bilinear switched systems realizing $\Phi$, but $\operatorname{dim} \Sigma_{m}=2$ and $\operatorname{dim} \Sigma_{c}=4$. Moreover, $\Sigma_{c}$ is quasi-minimal.

Example 2.3. Recall from Example 2.1 the definition of system $(\Sigma, \mu)$ and family of input-output maps $\Phi=\left\{f_{1}, f_{2}\right\}$. Let $L=\left\{\sigma_{1} \sigma_{2}^{k} \mid k \geq 0\right\}$. Let $\hat{f}_{i}, i=1,2$ be the restriction of $f_{i}, i=1,2$ to the set of admissible switching sequences $T L$. Consider $\hat{\Phi}=\left\{\hat{f}_{1}, \hat{f}_{2}\right\}$. Then $(\Sigma, \mu)$ is a realization of $\hat{\Phi}$ with constraint $L$. Recall from Example 2.2 the definition of the realization $\left(\Sigma_{m}, \mu_{m}\right)$. It follows that $\left(\Sigma_{m}, \mu_{m}\right)$ is also a realization of $\hat{\Phi}$ with constraint $L$, and $\operatorname{dim} \Sigma_{m}=2$. However, if we apply the minimization procedure for system with constrained switching described above, we obtained the realization $\left(\Sigma_{c}, \mu_{c}\right)$ of $\hat{\Phi}_{\text {; }}$ $\Sigma_{c}=\left(\mathcal{X}_{c}, \mathcal{U}, \mathcal{Y}, Q,\left\{\left(A_{q}^{c},\left\{B_{q, j}^{c}\right\}_{j=1,2, \ldots, m}, C_{q}^{c}\right) \mid q \in Q\right\}\right)$ where the state-space is $\mathcal{X}_{c}=\mathbb{R}^{4}$ and the matrices are defined as

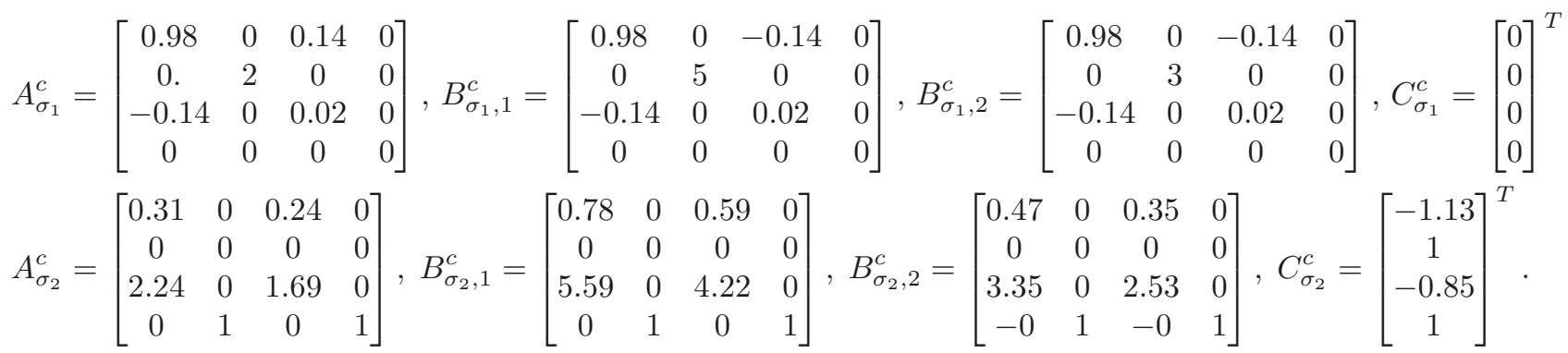

The map $\mu_{c}: \hat{\Phi} \rightarrow \mathbb{R}^{4}$ is defined as follows; $\mu_{c}\left(\hat{f}_{1}\right)=(-0.99,0,0,1.44,0)^{T}$ and $\mu_{c}\left(\hat{f}_{2}\right)=(0,1,0,0)^{T}$.

\subsection{Existence of a realization}

Below we present sufficient and necessary conditions for existence of a bilinear switched system realization. First, we present the notion of a generalized Fliess-series expansion, existence of which is a necessary condition for realizability by a bilinear switched system. We then formulate conditions for existence of a bilinear switched system realization, first for the case of arbitrary switching, and then for the case of constrained switching.

\subsubsection{Generalized Fliess series expansion}

Below we define the notion of a generalized Fliess-series expansion for a family of input-output maps. In the remainder of this section, $L \subseteq Q^{+}$is the set of admissible sequences of discrete modes and $\Phi \subseteq$ $F(P C(T, \mathcal{U}) \times T L, \mathcal{Y})$ is a set of input-output maps with the switching constraint L. Roughly speaking, existence of a generalized Fliess-series expansion for $\Phi$ means that every element of $\Phi$ is generated by a so called generalized convergent generating series (abbreviated GCGS). For the formal definition, we need the following.

Notation 2.6. Consider the infinite set $\widetilde{\Gamma}=Q \times \mathrm{Z}_{m}^{*}$. Following the convention of [6], Section 2.1, we denote by $\widetilde{\Gamma}^{*}$ the set of all finite words over $\widetilde{\Gamma}, \epsilon$ denotes the empty sequence over $\widetilde{\Gamma}$, and if $w, v \in \widetilde{\Gamma}^{*}$, vw denotes the concatenation of $v$ and $w$. In addition, $\widetilde{\Gamma}^{+}$denotes the set of all non-empty words over $\widetilde{\Gamma}$. Define the set

$$
J L=\left\{\left(q_{1}, w_{1}\right)\left(q_{2}, w_{2}\right) \ldots\left(q_{k}, w_{k}\right) \in \widetilde{\Gamma}^{*} \mid\left(q_{1}, w_{1}\right),\left(q_{2}, w_{2}\right), \ldots,\left(q_{k}, w_{k}\right) \in \widetilde{\Gamma}, k>0, q_{1} q_{2} \ldots q_{k} \in L\right\}
$$


That is, the set $J L$ consists of all sequences of pairs of discrete modes and sequences over $\mathrm{Z}_{m}$ such that the sequence of discrete modes belongs to $L$. Next, we define a relation $R^{*}$ on the words over $\widetilde{\Gamma}$, such that the following holds; if two words are related by $R^{*}$ and $c$ is a CGCS and it is defined for both of them, then $c$ should take the same value on both the words. This is needed to ensure that the input-output map $F_{c}$ generated by $c$ behaves like an input-output map of a switched system; it should give the same response for the pair of switching sequences $w(q, 0) v$ and $w v, w(q, t)(q, \hat{t}) v$ and $w(q, t+\hat{t}) v$. The formal definition of $R^{*}$ goes as follows.

Definition 2.4. Let $R \subseteq \widetilde{\Gamma}^{*} \times \widetilde{\Gamma}^{*}$ be the relation such that for all words $l, v \in \widetilde{\Gamma}^{*},(l, v) \in R$ if and only if there exist $h, s \in \widetilde{\Gamma}^{*}$ such that either

(1) $l=h\left(q, w_{1}\right)\left(q, w_{2}\right) s$ and $v=h\left(q, w_{1} w_{2}\right) s$ for some $w_{1}, w_{2} \in \mathrm{Z}_{m}^{*}$ and $q \in Q$; or

(2) $l=h\left(q_{1}, \epsilon\right)\left(q_{2}, w\right) s$ and $v=h\left(q_{2}, w\right) s$ for some $w \in \mathrm{Z}_{m}^{*}$, and $q_{1}, q_{2} \in Q$.

Let $R^{*} \subseteq \widetilde{\Gamma}^{*} \times \widetilde{\Gamma}^{*}$ be the symmetric, reflexive and transitive closure of $R$; i.e. $(l, v) \in R^{*}$ if and only if either $l=v$, or there exist $s_{0}, s_{1}, \ldots, s_{k+1} \in \widetilde{\Gamma}^{*}, k \geq 0$, such that for $i=0, \ldots, k$, either $\left(s_{i}, s_{i+1}\right) \in R$ or $\left(s_{i+1}, s_{i}\right) \in R$ and $s_{0}=l, s_{k+1}=v$.

The intuition behind the definition of $R$ is the following. Property (2) reflects the scenario when we stay in a discrete mode for zero time. Property (1) reflects the scenario when we jump to the same discrete mode. Recall that $\mathcal{U}=\mathbb{R}^{m}$ is the space of continuous inputs, $\mathcal{Y}=\mathbb{R}^{p}$ is the space of continuous outputs.

Definition 2.5 (generalized convergent generating series on JL). A map $c: J L \rightarrow \mathcal{Y}$ is called a generalized convergent generating series (abbreviated as GCGS) on $J L$ if the following conditions hold:

(1) For any two words $w$ and $v$ from $J L$, if $v$ and $w$ are related by $R^{*}$, i.e., if $(w, v) \in R^{*}$, then $c(w)=c(v)$.

(2) There exists $K, M>0$, such that for any $\left(q_{1}, w_{1}\right) \ldots\left(q_{k}, w_{k}\right) \in J L,\left(q_{1}, w_{1}\right), \ldots,\left(q_{k}, w_{k}\right) \in \widetilde{\Gamma}, k>0$,

$$
\left\|c\left(\left(q_{1}, w_{1}\right)\left(q_{2}, w_{2}\right) \ldots\left(q_{k}, w_{k}\right)\right)\right\|_{2}<K M^{\left|w_{1}\right|} \ldots M^{\left|w_{k}\right|} .
$$

Here $\|\cdot\|_{2}$ denotes the Euclidean norm on $\mathbb{R}^{p}$.

Notice that $c$ in the above definition is required to yield the same value for any two sequences related by $R^{*}$. Moreover, the values of $c$ are required to be bounded according to (2.19). The latter condition is needed in order to ensure the existence of the input-output map $F_{c}$ generated by $c$. The notion of GCGS is an extension of the classical notion of convergent generating series; if $L=Q^{+}$and $|Q|=1$, then a GCGS can be viewed as a convergent generating series from $[2,8]$. Next, we define the input-output map generated by a GCGS.

Definition 2.6. Let $c: J L \rightarrow \mathcal{Y}$ be a GCGS. Define the input-output map $F_{c}: P C(T, \mathcal{U}) \times T L \rightarrow \mathcal{Y}$ generated by $c$ as the follows. For each $u \in P C(T, \mathcal{U})$ and switching sequence $s=\left(q_{1}, t_{1}\right)\left(q_{2}, t_{2}\right) \ldots\left(q_{k}, t_{k}\right) \in T L$, $q_{1}, \ldots, q_{k} \in Q, t_{1}, \ldots, t_{k} \in T, k>0$,

$$
F_{c}(u, s)=\sum_{w_{1}, w_{2}, \ldots, w_{k} \in Z_{m}^{*}} c\left(\left(q_{1}, w_{1}\right)\left(q_{2}, w_{2}\right) \ldots\left(q_{k}, w_{k}\right)\right) V_{w_{1}, w_{2} \ldots, w_{k}}[u]\left(t_{1}, \ldots, t_{k}\right)
$$

Similarly to the classical case it can be shown that the series $F_{c}(u, s)$ is absolutely convergent and hence any convergent generating series $c$ defines an input-output map. The formal statement will be presented in Lemma A.1, Section A.1. Notice that the function $F_{c}$ is similar to the input-output function induced by a classical generating convergent series. In fact, similarly to the classical case, one can show that $F_{c}$ determines $c$ uniquely, i.e. if $F_{c}=F_{d}$ for two GCGS $c$ and $d$, then $c=d$, see Lemma A.2, Section A.1. Now we are ready to define the notion of a generalized Fliess-series expansion.

Definition 2.7 (generalized Fliess-series expansion). The family $\Phi$ is said to admit a generalized Fliess-series expansion if for each $f \in \Phi$ there exist a GCGS $c_{f}: J L \rightarrow \mathcal{Y}$ such that $c_{f}$ generates $f$, i.e. $F_{c_{f}}=f$.

From Lemma A.2, Appendix A.1 it follows that $\Phi$ admits at most one generalized Fliess-series expansion. Next, we describe the Fliess-series expansion of $\Phi$ if $\Phi$ is realizable by a bilinear switched system. 
Theorem 2.5 (realizability). Assume that $\Sigma$ is a bilinear switched system of the form (2.7) and let $\mu: \Phi \rightarrow \mathcal{X}$. Then $(\Sigma, \mu)$ is realization of $\Phi$ if and only if $\Phi$ has a generalized Fliess-series expansion such that for each $f \in \Phi$ the corresponding GCGS $c_{f}$ which generates $f$, i.e. $f=F_{c_{f}}$, is of the following form:

$$
c_{f}\left(\left(q_{1}, w_{1}\right)\left(q_{2}, w_{2}\right) \ldots\left(q_{k}, w_{k}\right)\right)=C_{q_{k}} B_{q_{k}, w_{k}} B_{q_{k-1}, w_{k-1}} \ldots B_{q_{1}, w_{1}} \mu(f)
$$

for each $\left(q_{1}, w_{1}\right) \ldots\left(q_{k}, w_{k}\right) \in J L$, where $q_{1}, \ldots, q_{k} \in Q, w_{1}, \ldots, w_{k} \in \mathrm{Z}_{m}^{*}, k>0$.

The proof of Theorem 2.5 can be found in Appendix A.

Example 2.4. The equations (2.12) in Example 2.1 define generalized convergent generating series. The series $c_{i}:\left(Q \times \mathrm{Z}_{2}^{*}\right)^{*} \ni s \mapsto c_{x_{0, i}}(s), i=1,2$ defines a generalized Fliess-series expansion for $f_{i}, i=1,2$. Recall the definition of the language $L$ from Example 2.3. The restriction of $c_{i}$ to $J L$ is a GCGS and it defines the generalized Fliess-series expansion of $\hat{f}_{i}, i=1,2$, where $\hat{f}_{i}, i=1,2$ is the restriction of $f_{i}, i=1,2$ to $T L$.

\subsubsection{Existence of a realization: arbitrary switching}

In this section we will present the main results on existence of a bilinear switched system realization for the case of arbitrary switching. For the rest of this section let $\Phi \subseteq F\left(P C(T, \mathcal{U}) \times(Q \times T)^{+}, \mathcal{Y}\right)$ be a set of input-output maps defined for arbitrary switching, and assume that $\Phi$ admits a generalized Fliess-series expansion. First, we define the concept of the Hankel-matrix of $\Phi$. To this end, we need additional notation and terminology. For each map $f$ from $\Phi$ denote by $c_{f}$ the GCGS which generates $f$. Since $L=Q^{+}$, $c_{f}$ is a map $c_{f}: \widetilde{\Gamma}^{+} \rightarrow \mathcal{Y}$.

Notation 2.7. Let $\Gamma=Q \times \mathrm{Z}_{m}$ be set of all pairs of discrete states and indices from $\mathrm{Z}_{m}$.

Notice that $\Gamma \subseteq \widetilde{\Gamma}$, but in contrast to $\widetilde{\Gamma}, \Gamma$ is finite. For each map $f \in \Phi$, discrete mode $q \in Q$, and sequence $w \in \Gamma^{*}$ define the vector $S_{f, q}(w) \in \mathbb{R}^{p}$ as follows:

$$
S_{f, q}(w)=c_{f}(w(q, \epsilon)) .
$$

Notice that $w(q, \epsilon)$ can naturally be interpreted as a sequence from $\widetilde{\Gamma}^{*}$, and hence the right-hand side of (2.22) is well-defined. The Hankel-matrix is constructed from the vectors $S_{f, q}(w)$ as follows.

Definition 2.8 (Hankel matrix). Assume that the cardinality of $Q$ is $N$, and consider the following enumeration of $Q$,

$$
Q=\left\{\sigma_{1}, \sigma_{2}, \ldots, \sigma_{N}\right\}
$$

The rows of the Hankel-matrix $H_{\Phi}$ of $\Phi$ are indexed by all the pairs $(v, i)$ where $i \in I=\{1,2, \ldots, N p\}$, and $v \in \Gamma^{*}$. The columns of $H_{\Phi}$ are indexed by all the pairs $(w, f)$ where $f \in \Phi$ and $w \in \Gamma^{*}$. That is, $H_{\Phi} \in \mathbb{R}^{\left(\Gamma^{*} \times I\right) \times\left(\Gamma^{*} \times \Phi\right)}$. For each input-output map $f \in \Phi$, words $w, v \in \Gamma^{*}$, and for each $i \in\{1,2, \ldots, N p\}$ of the form $i=p K+r$ for some $K=0,1, \ldots, N-1$ and $r=1, \ldots, p$, the entry $\left(H_{\Phi}\right)_{(v, i),(w, f)}$ of $H_{\Phi}$ indexed by the row index $(v, i)$ and column index $(w, f)$ is of the form

$$
\left(H_{\Phi}\right)_{(v, i),(w, f)}=\left(S_{f, \sigma_{K+1}}(w)\right)_{r}
$$

where $\left(S_{f, \sigma_{K+1}}(w)\right)_{r}$ denotes the $r$ th entry of the vector $S_{f, \sigma_{K+1}}(w) \in \mathbb{R}^{p}$. Following the convention of [6], Section 2.1, the rank of $H_{\Phi}$, denoted by rank $H_{\Phi}$, is the dimension of the linear space spanned by the columns of $H_{\Phi}$.

I.e., the block $\left(\left(H_{\Phi}\right)_{(v, i),(w, f)}\right)_{i=1, \ldots, p N}=\left[\begin{array}{llll}\left(H_{\Phi}\right)_{(v, 1),(w, f)} & \left(H_{\Phi}\right)_{(v, 2),(w, f)} \quad \ldots \quad\left(H_{\Phi}\right)_{(v, N p),(w, f)}\end{array}\right]^{T} \in \mathbb{R}^{p N \times 1}$ of $H_{\Phi}$ formed by the entries indexed with the column index $(w, f)$ and row indices $(v, i), i=1,2, \ldots, p N$ equals

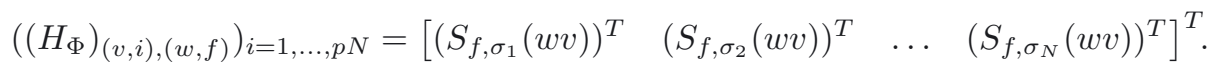


Theorem 2.6 (existence of a realization: arbitrary switching). The family $\Phi$ has a realization by a bilinear switched system, if and only if $\Phi$ has a generalized Fliess-series expansion and rank $H_{\Phi}<+\infty$, i.e. the rank of the Hankel-matrix is finite.

The proof of Theorem 2.6 can be found in Section 3.1.

\subsubsection{Existence of a realization: constrained switching}

In this section we will present the main result on existence of a realization by a bilinear switched system for the case of constrained switching. In the rest of the section, let $L \subseteq Q^{+}$be a subset of admissible sequences of discrete modes and let $\Phi \subseteq F(P C(T, \mathcal{U}) \times T L, \mathcal{Y})$ be a family of input-output maps with the switching constraint $L$. We assume that $\Phi$ admits a generalized Fliess-series expansion. Similarly to the case of arbitrary switching, we will use the GCGS $c_{f}, f \in \Phi$ for defining the Hankel-matrix $H_{\Phi}$ of $\Phi$. The only problem is that $c_{f}(s)$ is not defined on the whole $\widetilde{\Gamma}^{+}$. We solve this problem by assuming that the value of $c_{f}$ is zero for those sequences for which we cannot deduce any information. Then we repeat the definition of the Hankel-matrix presented for the case of arbitrary switching. The details are as follows.

Recall the definition of the relation $R^{*} \subseteq \widetilde{\Gamma}^{*} \times \widetilde{\Gamma}^{*}$ from Definition 2.4 and recall from (2.18) the definition of the set $J L$. Define the set $\widetilde{J L} \subseteq \widetilde{\Gamma}^{*}$ as the set of all those words $s \in \widetilde{\Gamma}^{*}$ such that for some $w \in J L,(w, s) \in R^{*}$, i.e.

$$
\widetilde{J L}=\left\{s \in \widetilde{\Gamma}^{*} \mid \exists w \in J L:(w, s) \in R^{*}\right\} .
$$

The set $\widetilde{J L}$ contains all those sequences in $\widetilde{\Gamma}^{*}$ for which we can derive some information based on the values of a GCGS on $J L$. Recall the definition of $S_{f, q}$ from (2.22). Below we present a counterpart of $S_{f, q}$, denoted by $T_{f, q}$, for the case of constrained switching. For each discrete mode $q \in Q$, input-output map $f \in \Phi$ and for each sequence $s \in \Gamma^{*}$, where as in Notation 2.7, $\Gamma=Q \times \mathrm{Z}_{m}$, define $T_{f, q}(s) \in \mathbb{R}^{p}$ as

$$
T_{f, q}(s)=\left\{\begin{aligned}
c_{f}(w) & \text { if } s(q, \epsilon) \in \widetilde{J L} \\
0 & \text { otherwise. }
\end{aligned} \text { and } w \in J L \quad \text { and } \quad(s(q, \epsilon), w) \in R^{*} .\right.
$$

The well-definedness of $T_{f, q}(s)$ will be shown in Section 3.2. The intuition behind the definition of $T_{f, q}(s)$ is the following. We store in $T_{f, q}(s)$ the values of all those $c_{f}(w)$ which show up in the generalized Fliess-series expansion of $f(u, v)$, for some switching sequence $v \in T L$ such that $v$ ends with discrete mode $q$. For all the other sequences, we set $T_{f, q}(s)$ to zero. If $L=Q^{+}$, then $S_{f, q}(s)=T_{f, q}(s)$ for all $s \in \Gamma^{*}$. The definition of the Hankel-matrix $H_{\Phi}$ is the same as for the case or arbitrary switching if we replace $S_{f, q}$ with $T_{f, q}$.

Definition 2.9 (Hankel-matrix). Fix the enumeration of $Q$ as in (2.23), i.e. $|Q|=N$ and $Q=\left\{\sigma_{1}, \sigma_{2}, \ldots, \sigma_{N}\right\}$. The rows of the Hankel-matrix $H_{\Phi}$ are indexed by pairs $(v, i)$ where $i \in I=\{1,2, \ldots, N p\}$ and $v \in \Gamma^{*}$. The columns of $H_{\Phi}$ are indexed by pairs $(w, f)$ where $f \in \Phi$ and $w \in \Gamma^{*}$. $H_{\Phi} \in \mathbb{R}^{\left(\Gamma^{*} \times I\right) \times\left(\Gamma^{*} \times \Phi\right)}$. For any map $f \in \Phi$, words $w, v \in \Gamma^{*}$, and for any $i=1, \ldots, N p$ of the form $i=K p+r$ with $K=0, \ldots, N-1$ and $r=1, \ldots, p$, the entry $\left(H_{\Phi}\right)_{(v, i),(w, f)}$ with the row index $(v, i)$ and column index $(w, f)$ is

$$
\left(H_{\Phi}\right)_{(v, i),(w, f)}=\left(T_{f, \sigma_{K+1}}(w v)\right)_{r}
$$

where $\left(T_{f, \sigma_{K+1}}(w v)\right)_{r}$ denotes the $r$ th entry of the vector $T_{f, \sigma_{K+1}}(w v) \in \mathbb{R}^{p}$. Following the convention of [6], Section 2.1, the rank of $H_{\Phi}$, denoted by rank $H_{\Phi}$, is the dimension of the linear space spanned by the columns of $H_{\Phi}$.

Theorem 2.7 (existence of a realization: constrained switching). If $\Phi$ has a generalized Fliess-series expansion and the rank of the Hankel-matrix of $\Phi$ is finite, i.e. rank $H_{\Phi}<+\infty$, then $\Phi$ has a realization by a bilinear switched system. If $L$ is regular, then the above condition is also sufficient, i.e. if $L$ is regular, $\Phi$ has a generalized Fliess-series expansion and rank $H_{\Phi}<+\infty$, then $\Phi$ has a bilinear switched system realization.

The proof of Theorem 2.7 will be presented in Section 3.2. 
Remark 2.5 (construction of a realization from the Hankel-matrix). The proofs of Theorems 2.6 and 2.7 also yield a procedure for constructing a bilinear switched system realization of $\Phi$ from the columns of the Hankel-matrix $H_{\Phi}$ both for the case of arbitrary and constrained switching. For the details see Section 3.

\section{Proof of the MAin ReSults}

\subsection{Proof of the main results: arbitrary switching}

\subsubsection{Existence of a realization: proof of Theorem 2.6}

In the sequel, unless stated otherwise, $\Phi \subseteq F\left(P C(T, \mathcal{U}) \times(Q \times T)^{+}, \mathcal{Y}\right)$ denotes a family of input-output maps with arbitrary switching and it is assumed that $\Phi$ admits generalized Fliess-series expansion. The proof is based on the relationship between bilinear switched system realizations of $\Phi$ and rational representations of the family of formal power series $\Psi_{\Phi}$ to be defined below. More precisely, the family $\Psi_{\Phi}$ will be such that $\Phi$ is realizable by a bilinear switched system if and only if $\Psi_{\Phi}$ is rational. Recall from Section $2.4,(2.22)$ the definition of the the vector $S_{f, q}(w)$ defined for all discrete modes $q \in Q$, maps $f$ from $\Phi$ and words $w \in \Gamma^{*}$. It is easy to see that the $\operatorname{map} S_{f, q}: \Gamma^{*} \ni w \mapsto S_{f, q}(w) \in \mathbb{R}^{p}$ can be viewed as a formal power series, i.e. $S_{f, q} \in \mathbb{R}^{p} \ll \Gamma^{*} \gg$. Recall the enumeration $Q=\left\{\sigma_{1}, \ldots, \sigma_{N}\right\}$ of $Q$ already defined in (2.23). Define the formal power series $S_{f} \in \mathbb{R}^{N p} \ll \Gamma^{*} \gg$ such that for all $w \in \Gamma^{*}$,

$$
S_{f}(w)=\left[\begin{array}{llll}
\left(S_{f, \sigma_{1}}(w)\right)^{T} & \left(S_{f, \sigma_{2}}(w)\right)^{T} \quad \ldots \quad\left(S_{f, \sigma_{N}}(w)\right)^{T}
\end{array}\right]^{T} .
$$

That is, $S_{f}$ is obtained by "stacking up" vertically the values of $S_{f, q}$ for all $q \in Q$. Define the family of formal power series $\Psi_{\Phi}$ associated with $\Phi$ as follows

$$
\Psi_{\Phi}=\left\{S_{f} \in \mathbb{R}^{N p} \ll \Gamma^{*} \gg \mid f \in \Phi\right\} .
$$

Note that $\Psi_{\Phi}$ is indexed by the input-output maps from $\Phi$.

Remark 3.1 (equivalence of Hankel-matrices). It is easy to see that the Hankel-matrix $H_{\Psi_{\Phi}}$ of the family of formal power series $\Psi_{\Phi}$ is identical to the Hankel-matrix $H_{\Phi}$ of $\Phi$ as defined in Definition 2.8, Section 2.4, and their ranks are identical too.

We will associate a rational representation $R_{\Sigma, \mu}$ with any bilinear switched realization $(\Sigma, \mu)$ in such a way that $R_{\Sigma, \mu}$ is a representation of $\Psi_{\Phi}$ if and only if $(\Sigma, \mu)$ is a realization of $\Phi$. Conversely, we want to construct a bilinear switched system realization $\left(\Sigma_{R}, \mu_{R}\right)$ from any rational representation $R$, such that $R$ is a representation of $\Psi_{\Phi}$ if and only if $\left(\Sigma_{R}, \mu_{R}\right)$ is a realization of $\Phi$.

Below we will present the precise constructions for both cases. These constructions will also be used for the case of constrained switching. For this reason, in the constructions below we will allow $\Phi$ to be a family of input-output maps with a switching constraint $L \subseteq Q^{+}$, i.e. $\Phi \subseteq F(P C(T, \mathcal{U}) \times T L, \mathcal{Y})$.

Construction 3.1 (representation associated with a bilinear switched system realization). Let $\Phi \subseteq$ $F(P C(T, \mathcal{U}) \times T L, \mathcal{Y})$ with $L \subseteq Q^{+}$, and let $\Sigma$ be a bilinear switched system of the form (2.7) and assume that $\mu: \Phi \rightarrow \mathcal{X}$. Define the representation $R_{\Sigma, \mu}$ associated with the realization $(\Sigma, \mu)$ of $\Phi$ as the representation

$$
R_{\Sigma, \mu}=\left(\mathcal{X},\left\{B_{(q, j)}\right\}_{(q, j) \in \Gamma}, I, \widetilde{C}\right) .
$$

The state-space $\mathcal{X}=\mathbb{R}^{n}$ coincides with that of $\Sigma$. For each discrete mode $q \in Q$ and index $j=1, \ldots, m$, let $B_{(q, j)}=B_{q, j}$, and let $B_{(q, 0)}=A_{q}$. Let $\sigma_{1}, \sigma_{2}, \ldots, \sigma_{N}$ be the discrete modes in the enumeration of $Q$ from (2.23). Then $\widetilde{C}$ is obtained by vertically "stacking up" the matrices $C_{\sigma_{1}}, C_{\sigma_{2}}, \ldots, C_{\sigma_{N}}$ in this order, that is,

$$
\widetilde{C}=\left[\begin{array}{llll}
C_{\sigma_{1}}^{T} & C_{\sigma_{2}}^{T} & \ldots & C_{\sigma_{N}}^{T}
\end{array}\right]^{T} .
$$

Finally, the family of initial states $I=\left\{I_{f} \in \mathcal{X} \mid f \in \Phi\right\}$ is defined by $I_{f}=\mu(f) \in \mathcal{X}$ for all $f \in \Phi$. 
The intuition behind the definition of $R_{\Sigma, \mu}$ is the following. If $L=Q^{+}$, then we want $R_{\Sigma, \mu}$ to be a representation of $\Psi_{\Phi}$, if $(\Sigma, \mu)$ is a realization of $\Phi$. We know that the rows of $S_{f}$ are $S_{f, \sigma_{1}}, S_{f, \sigma_{2}}, \ldots, S_{f, \sigma_{N}}$. Later we show that if $\Phi$ is realized by $(\Sigma, \mu)$ for each $i=1,2, \ldots, p$, then the values of $S_{f, \sigma_{i}}$ can be expressed as products of the system matrices $B_{q, j}$ of $\Sigma$ multiplied from the right by $\mu(f)$ and from the left by $C_{\sigma_{i}}$. By interpreting the "stacking up" of the matrices $C_{\sigma_{i}}$ as the readout matrix, interpreting the matrices $B_{q, j}$ as transition matrices, and interpreting the state $\mu(f)$ as initial state, we get a rational representation of $\Psi_{\Phi}$.

We would like to carry out the converse step, that is, we would like to build a bilinear switched system representation $\left(\Sigma_{R}, \mu_{R}\right)$ from a representation $R$.

Construction 3.2 (bilinear switched system realization associated with a rational representation). Denote by $L$ is the set of admissible sequences of discrete modes, i.e. $L \subseteq Q^{+}$and let $\Phi \subseteq F(P C(T, \mathcal{U}) \times T L, \mathcal{Y})$ be a family of input-output maps with the switching constraint $L$. Let

$$
R=\left(\mathcal{X},\left\{M_{(q, j)}\right\}_{(q, j) \in \Gamma}, I, \widetilde{C}\right)
$$

be a rational representation such that the family of initial states $I=\left\{I_{f} \in \mathcal{X} \mid f \in \Phi\right\}$ is indexed by the elements of $\Phi$ and and the range of the readout map $\widetilde{C}$ lies in $\mathbb{R}^{p N}$, i.e. $R$ is an $p N$ - $\Phi$ representation. If $\mathcal{X}$ is not $\mathbb{R}^{n}$, then replace the rational representation $R$ by its isomorphic copy $T R$ described in [6], Remark 12, such that the state-space of $T R$ is $\mathbb{R}^{n}, n=\operatorname{dim} \mathcal{X}$. In the rest of the construction we assume that that the state-space of $R$ is $\mathcal{X}=\mathbb{R}^{n}$. Hence the state-transition maps $M_{(q, j)}, q \in Q, j=1, \ldots, m$ can be viewed as $n \times n$ matrices, and the readout map $\widetilde{C}$ can be viewed as a $p N \times n$ matrix. Define the bilinear switched system realization $\left(\Sigma_{R}, \mu_{R}\right)$ associated with $R$ as follows. Let $\Sigma_{R}$ be of the form (2.7). The state space of $\Sigma_{R}$ coincides with that of the representation $R$. The system matrices of $\Sigma_{R}$ are derived from those of $R$ as follows. For each discrete mode $q \in Q, A_{q}=M_{(q, 0)}$ and $B_{q, j}=M_{(q, j)}, j=1, \ldots, m$. The matrices $C_{q}, q \in Q$ are defined as follows. Consider the enumeration $Q=\left\{\sigma_{1}, \sigma_{2}, \ldots, \sigma_{N}\right\}$ from $(2.23)$. For any $q=\sigma_{i}, i=1,2, \ldots, N, r=1, \ldots, p$, the $r$ th row of the matrix $C_{q}$ equals the $(i-1) p+r$ th row of $\widetilde{C}$, i.e.

$$
\widetilde{C}=\left[\begin{array}{llll}
C_{\sigma_{1}}^{T} & C_{\sigma_{2}}^{T} & \ldots & C_{\sigma_{N}}^{T}
\end{array}\right]^{T} .
$$

Finally, define the map $\mu_{R}: \Phi \rightarrow \mathcal{X}$ as follows; $\mu_{R}(f)=I_{f} \in \mathcal{X}$ for all $f \in \Phi$.

Intuitively, the matrices of $\left(\Sigma_{R}, \mu_{R}\right)$ are defined in such a way that if $R$ is a representation of $\Psi_{\Phi}$ then $(2.21)$ of Theorem 2.5 holds for $\left(\Sigma_{R}, \mu_{R}\right)$, i.e. $\left(\Sigma_{R}, \mu_{R}\right)$ is a realization of $\Phi$. It is easy to see that Constructions 3.1 and 3.2 commute in the following sense.

Lemma 3.1. $R_{\Sigma_{R}, \mu_{R}}$ is isomorphic to $R$ and $\left(\Sigma_{R_{\Sigma, \mu}}, \mu_{R_{\Sigma, \mu}}\right)=(\Sigma, \mu)$. If the state-space of $R$ is of the form $\mathbb{R}^{n}$, then $R_{\Sigma_{R}, \mu_{R}}=R$.

Theorem 3.1 (correspondence between rationality and realizability). Assume that $\Phi$ is a family of input-output maps defined for arbitrary switching, and assume that $\Phi$ admits a generalized Fliess-series expansion. $(\Sigma, \mu)$ is a realization of $\Phi$ if and only if $R_{\Sigma, \mu}$ is a representation of $\Psi_{\Phi}$. Conversely, $R$ is a representation of $\Psi_{\Phi}$ if and only if $\left(\Sigma_{R}, \mu_{R}\right)$ is a realization of $\Phi$.

Notice that if $R$ is a representation if $\Psi_{\Phi}$, then a suitable isomorphic copy of $R$ satisfies the condition of Construction 3.2, and hence $\left(\Sigma_{R}, \mu_{R}\right)$ is defined. We present the proof of Theorem 3.1 at the end of this section.

Proof of Theorem 2.6. By Theorem 2.5 if $(\Sigma, \mu)$ is a bilinear switched system realization of $\Phi$, then $\Phi$ has a generalized Fliess-series expansion, and from Theorem 3.1 we get that then $\Psi_{\Phi}$ is rational. Conversely, if $\Phi$ has a generalized Fliess-series expansion and $\Psi_{\Phi}$ is rational, then by choosing an appropriate representation $R$ of $\Psi_{\Phi}$, from Theorem 3.1 it follows that $\left(\Sigma_{R}, \mu_{R}\right)$ is a realization of $\Phi$. By [6], Theorem 4.1 and Remark 3.1, $\Psi_{\Phi}$ is rational if and only if rank $H_{\Psi_{\Phi}}=\operatorname{rank} H_{\Phi}<+\infty$. 
We devote the rest of the section to proving Theorem 3.1. To this end, we need a number of technical results. Define the map $\phi: \widetilde{\Gamma} \rightarrow \Gamma^{*}$ by $\phi((q, w))=\left(q, j_{1}\right)\left(q, j_{2}\right) \ldots\left(q, j_{k}\right)$ where $w=j_{1} j_{2} \ldots j_{k} \in Z_{m}^{*}, j_{1}, j_{2}, \ldots, j_{k} \in$ $\mathrm{Z}_{m}, k>0$, and $\phi((q, \epsilon))=\epsilon$. The map $\phi$ can be extended to a monoid morphism ${ }^{3} \phi: \widetilde{\Gamma}^{*} \rightarrow \Gamma^{*}$ given by

$$
\phi\left(\left(q_{1}, w_{1}\right)\left(q_{2}, w_{2}\right) \ldots\left(q_{k}, w_{k}\right)\right)=\phi\left(\left(q_{1}, w_{1}\right)\right) \phi\left(\left(q_{2}, w_{2}\right)\right) \ldots \phi\left(\left(q_{k}, w_{k}\right)\right)
$$

for each $\left(q_{1}, w_{1}\right),\left(q_{2}, w_{2}\right), \ldots,\left(q_{k}, w_{k}\right) \in \widetilde{\Gamma}, k \geq 0$. If $k=0$, then we set $\phi(\epsilon)=\epsilon$. That is, $\phi$ simply "unwraps" every element of the form $(q, w)$ into a sequence of pairs $\left(q, j_{1}\right)\left(q, j_{2}\right) \ldots\left(q, j_{k}\right)$ where the second component of each pair consists of one single letter. It is also clear that any element of $\Gamma$ can be thought of as an element of $\widetilde{\Gamma}$. It is also easy to see that $\phi(w)=w$ for any word $w$ from $\Gamma^{*}$. Below we state a number of technical lemmas which describe the relationship between $\phi$ and the relation $R^{*}$ from Definition 2.4.

Lemma 3.2. If $(l, v) \in R^{*}$, then $\phi(l)=\phi(v)$ for any $l, v \in \widetilde{\Gamma}^{*}$.

Lemma 3.3. For any $v \in \widetilde{\Gamma}^{*}$ and for any $q \in Q,(v(q, \epsilon), \phi(v)(q, \epsilon)) \in R^{*}$.

The proof of Lemmas 3.2 and 3.3 can be found in Appendix A.3.

Lemma 3.4. For any input-output map $f \in \Phi$, word $w \in \widetilde{\Gamma}^{*}$, and discrete mode $q \in Q, c_{f}(w(q, \epsilon))=S_{f, q}(\phi(w))$.

Proof of Lemma 3.4. Lemma 3.3 implies that for all $q \in Q,(w(q, \epsilon), \phi(w)(q, \epsilon)) \in R^{*}$. Since $c_{f}$ satisfies Part (1) of Definition 2.5, it follows that $c_{f}(w(q, \epsilon))=c_{f}(\phi(w)(q, \epsilon))$. But from (2.22) it follows that $S_{f, q}(\phi(w))=$ $c_{f}(\phi(w)(q, \epsilon))$, hence we get the statement of the lemma.

Lemma 3.5. Assume that $\Phi$ is a family of input-output maps with the switching constraint $L \subseteq Q^{+}, \Sigma$ is of the form (2.7), $\mu: \Phi \rightarrow \mathcal{X}$, and $R_{\Sigma, \mu}$ is of the form (3.3). For each sequence $q_{1}, \ldots, q_{k} \in Q, w_{1}, \ldots, w_{k} \in \mathrm{Z}_{m}^{*}$, $k \geq 0$,

$$
B_{q_{1}, w_{1}} B_{q_{2}, w_{2}} \ldots B_{q_{k}, w_{k}}=B_{\phi\left(\left(q_{1}, w_{1}\right)\left(q_{2}, w_{2}\right) \ldots\left(q_{k}, w_{k}\right)\right)} .
$$

Here, on the right-hand side we used [6], Notation 4.1, for products of state-transition matrices of $R_{\Sigma, \mu}$.

Proof. For $j_{1}, \ldots, j_{l} \in \mathrm{Z}_{m}, l>0$,

$$
B_{q, j_{1} j_{2} \ldots j_{l}}=B_{q, j_{l}} B_{q, j_{l-1}} \ldots B_{q, j_{1}}=B_{\left(q, j_{l}\right)} B_{\left(q, j_{l-1}\right)} \ldots B_{\left(q, j_{1}\right)}=B_{\phi\left(q, j_{1} j_{2} \ldots j_{l}\right)}
$$

By repeatedly applying (3.6), we get the statement of the lemma.

Proof of Theorem 3.1. It is enough prove Part (a). Part (b) follows from Part (a) by using that fact that $R_{\Sigma_{R}, \mu_{R}}$ is isomorphic to $R$, established in Lemma 3.1. First we show that if $(\Sigma, \mu)$ is a realization of $\Phi$, then $R_{\Sigma, \mu}$ is a representation of $\Psi_{\Phi}$. Assume that $(\Sigma, \mu)$ is a realization of $\Phi$ and assume that $\Sigma$ is of the form (2.7).

For each $s=\left(q_{1}, j_{1}\right) \ldots\left(q_{k}, j_{k}\right) \in \Gamma^{*}, k \geq 0, q_{1}, \ldots, q_{k} \in Q, j_{1}, \ldots, j_{k} \in \mathrm{Z}_{m}$, apply $(2.21)$ to $s(q, \epsilon) \in \widetilde{\Gamma}^{*}$. Notice that $s=\phi(s), B_{q, \epsilon}=I_{n}$ and use Lemma 3.4, formula (3.5), and construction of $R_{\Sigma, \mu}$. Then,

$$
S_{f, q}(s)=S_{f, q}(\phi(s))=c_{f}(s(q, \epsilon))=C_{q} B_{q_{k}, j_{k}} \ldots B_{q_{1}, j_{1}} \mu(f)=C_{q} B_{\phi(s)} I_{f}=C_{q} B_{s} I_{f} .
$$

By stacking up $S_{f, q}(s)$ and taking into account the definition of $S_{f}(s)$ from (3.1) and the definition the readout matrix $\widetilde{C}$, we get that for any $s \in \Gamma$ and $f \in \Phi, S_{f}(s)=\widetilde{C} B_{s} I_{f}$, i.e. $R_{\Sigma, \mu}$ is a representation of $\Psi_{\Phi}$.

Conversely, assume that $R_{\Sigma, \mu}$ is a representation of $\Psi$. This is equivalent to saying that for any discrete mode $q \in Q$, word $s \in \Gamma^{*}$ and map $f \in \Phi, S_{f, q}(s)=C_{q} B_{s} I_{f}$. Consider the sequence $v=\left(q_{1}, w_{1}\right)\left(q_{2}, w_{2}\right) \ldots\left(q_{k}, w_{k}\right) \in$ $\widetilde{\Gamma}^{*}, q_{1}, \ldots, q_{k} \in Q, w_{1}, \ldots, w_{k} \in \mathrm{Z}_{m}^{*}, k>0$. Notice that $\left(v\left(q_{k}, \epsilon\right), v\right) R^{*}$, hence, $c_{f}\left(v\left(q_{k}, \epsilon\right)\right)=c_{f}(v)$.

\footnotetext{
${ }^{3}$ Recall that a monoid is a semigroup with identity. A monoid morphism is a map between two monoids which preserves the multiplication and maps the identity element to the identity element.
} 
By combining the remarks above Lemma 3.4, (3.5) and the definition of $I_{f}$, we get that

$$
c_{f}(v)=c_{f}\left(v\left(q_{k}, \epsilon\right)\right)=S_{f, q_{k}}(\phi(v))=C_{q_{k}} B_{\phi(v)} I_{f}=C_{q_{k}} B_{q_{k}, w_{k}} B_{q_{k-1}, w_{k-1}} \ldots B_{q_{1}, w_{1}} \mu(f) .
$$

By Theorem 2.5, (3.8) is equivalent to $(\Sigma, \mu)$ being a realization of $\Phi$.

\subsubsection{Minimality: proof of Theorem 2.3}

We reduce minimality of a bilinear switched system realization to minimality of the associated rational representation. To this end, we first relate observability and semi-reachability of a bilinear switched system realization with observability and reachability of the corresponding rational representation.

Lemma 3.6. Assume that $\Phi$ is a family of input-output maps with the switching constraint $L \subseteq Q^{+}, \Sigma$ is of the form (2.7), and $\mu: \Phi \rightarrow \mathcal{X}$. Then $(\Sigma, \mu)$ is observable if and only if the associated representation $R_{\Sigma, \mu}$ is observable. $(\Sigma, \mu)$ is semi-reachable from $\operatorname{Im} \mu$ if and only if $R_{\Sigma, \mu}$ is reachable. Conversely, if $R$ is a $p N-\Phi$ representation, then $R$ is reachable or observable, if and only if $\left(\Sigma_{R}, \mu_{R}\right)$ is semi-reachable respectively observable.

Proof. Since $R$ is isomorphic to $R_{\Sigma_{R}, \mu_{R}}$, it is enough to prove the first statement of the lemma. From (3.5) in the proof of Theorem 3.1 we get that for any $\left(q_{1}, w_{1}\right), \ldots,\left(q_{k}, w_{k}\right) \in \widetilde{\Gamma}, k>0$,

$$
\operatorname{ker} \widetilde{C} B_{\phi\left(\left(q_{k}, w_{k}\right)\right)} B_{\phi\left(\left(q_{k-1}, w_{k-1}\right)\right)} \ldots B_{\phi\left(\left(q_{1}, w_{1}\right)\right)}=\bigcap_{q \in Q} \operatorname{ker} C_{q} B_{q_{k}, w_{k}} B_{q_{k-1}, w_{k-1}} \ldots B_{q_{1}, w_{1}} .
$$

Recall from Theorem 2.2 the definition of $O_{\Sigma}$. Since $B_{q, \epsilon}=I_{n}$ and thus $C_{q}=C_{q} B_{q, \epsilon}$, (3.9) implies that $O_{\Sigma} \subseteq O_{R_{\Sigma, \mu}}$. On the other hand, (3.9) and the definition of $O_{R_{\Sigma, \mu}}$ imply that $O_{R_{\Sigma, \mu}} \subseteq O_{\Sigma}$.

Recall from (2.13), Theorem 2.2 the representation of the set $W(\operatorname{Im} \mu)$. Notice that $\operatorname{Im} \mu=\{\mu(f) \mid f \in \Phi\}=$ $\left\{I_{f} \mid f \in \Phi\right\}$. Hence, using (3.5) we get that $W(\operatorname{Im} \mu)=W_{R_{\Sigma, \mu}}$. Then the lemma follows from Theorem 2.2 and the definition of observability and reachability for representations.

Lemma 3.7. Assume that $\Phi$ is a family of input-output maps with arbitrary switching. Note that $\operatorname{dim} R=$ $\operatorname{dim} \Sigma_{R}$, and $\operatorname{dim} \Sigma=\operatorname{dim} R_{\Sigma, \mu}$. Hence, if $R$ is a minimal representation of $\Psi_{\Phi}$ then $\left(\Sigma_{R}, \mu_{R}\right)$ is a minimal realization of $\Phi$. Conversely, if $(\Sigma, \mu)$ is a minimal realization of $\Phi$, then $R_{\Sigma, \mu}$ is a minimal representation of $\Psi_{\Phi}$.

Next, we present the relationship between representation morphisms and bilinear switched system morphisms.

Lemma 3.8. Assume that $\Phi$ is a family of input-output maps with the switching constraint $L \subseteq Q^{+},(\Sigma, \mu)$ and $\left(\Sigma^{\prime}, \mu^{\prime}\right)$ are bilinear switched system realizations, and $\Phi$ is the domain of $\mu$ and $\mu^{\prime}$. Then $T:(\Sigma, \mu) \rightarrow\left(\Sigma^{\prime}, \mu^{\prime}\right)$ is a bilinear switched system morphism if and only if $T: R_{\Sigma, \mu} \rightarrow R_{\Sigma^{\prime}, \mu^{\prime}}$ is a representation morphism.

Proof. Assume that $\Sigma$ is of the form $(2.7)$ and $\Sigma^{\prime}=\left(\mathcal{X}^{\prime}, \mathcal{U}, \mathcal{Y}, Q,\left\{\left(A_{q}^{\prime},\left\{B_{q, j}^{\prime}\right\}_{j=1,2, \ldots, m}, C_{q}^{\prime}\right) \mid q \in Q\right\}\right)$. Assume that $R_{\Sigma, \mu}=\left(\mathcal{X},\left\{B_{(q, j)}\right\}_{(q, j) \in \Gamma}, I, \widetilde{C}\right)$ and $R_{\Sigma^{\prime}, \mu^{\prime}}=\left(\mathcal{X}^{\prime},\left\{B_{(q, j)}^{\prime}\right\}_{(q, j) \in \Gamma}, I^{\prime}, \widetilde{C}^{\prime}\right)$, where $I^{\prime}=\left\{I_{f}^{\prime} \in \mathcal{X}^{\prime} \mid f \in \Phi\right\}$. Then $T$ is a bilinear switched system morphism if and only if for each discrete mode $q \in Q$

$$
T A_{q}=A_{q}^{\prime} T \quad C_{q}=C_{q}^{\prime} T \quad T B_{q, j}=B_{q, j}^{\prime} T \quad T \mu(f)=\mu^{\prime}(f)
$$

where $j=1,2, \ldots, m$ and $f \in \Phi$. This is equivalent to $T B_{(q, j)}=B_{(q, j)}^{\prime} T$ for each $j \in \mathrm{Z}_{m}, T I_{f}=T \mu(f)=$ $\mu^{\prime}(f)=I_{f}^{\prime}$ for each input-output map $f \in \Phi$ and $\widetilde{C}=\left[\begin{array}{lll}C_{\sigma_{1}}^{T} & \ldots & C_{\sigma_{N}}^{T}\end{array}\right]^{T}=\left[\begin{array}{lll}\left(C_{\sigma_{1}}^{\prime} T\right)^{T} & \ldots & \left(C_{\sigma_{N}}^{\prime} T\right)^{T}\end{array}\right]^{T}=\widetilde{C}^{\prime} T$. The equalities above are equivalent to $T$ being a representation morphism.

Proof of Theorem 2.3. $\left(\Sigma_{\min }, \mu_{\min }\right)$ is a minimal realization if and only if that $R_{\min }=R_{\Sigma_{\min }, \mu_{\min }}$ is minimal representation, that is, by [6], Theorem 4.2, $R_{\min }$ is reachable and observable. By Lemma 3.6 the latter is 
equivalent to $\left(\Sigma_{\min }, \mu_{\min }\right)$ being semi-reachable from $\operatorname{Im} \mu$ and observable. That is, we get that (i) $\Longleftrightarrow$ (ii). By [6], Theorem 4.2, a representation $R_{\min }$ is minimal if and only if $\operatorname{dim} \Sigma_{\min }=\operatorname{dim} R_{\min }=\operatorname{rank} H_{\Phi \Psi}=$ rank $H_{\Phi}$. Here we used Remark 3.1 to derive the last equality. That is, we showed that (i) $\Longleftrightarrow$ (iii). To show that (i) $\Longleftrightarrow$ (iv), notice that $\left(\Sigma_{\min }, \mu_{\min }\right)$ is a minimal realization if and only if $R_{\Sigma_{\min }, \mu_{\min }}$ is a minimal representation. By [6], Theorem 4.2, $R_{\min }$ is minimal if and only if for any reachable representation $R$ there exists a surjective representation morphism $T: R \rightarrow R_{\min }$. It means that $\left(\Sigma_{\min }, \mu_{\min }\right)$ is minimal if and only if for any reachable representation $R$ of $\Psi_{\Phi}$ there exists a surjective representation morphism $T: R \rightarrow R_{\Sigma_{\min }, \mu_{\min }}$. But any reachable representation $R$ gives rise to a semi-reachable realization of $\Phi$ and vice versa. That is, we get that $\left(\Sigma_{\min }, \mu_{\min }\right)$ is minimal if and only if for any realization $\left(\Sigma^{\prime}, \mu^{\prime}\right)$ of $\Phi$ such that $\left(\Sigma^{\prime}, \mu^{\prime}\right)$ is semi-reachable from $\operatorname{Im} \mu$ there exists a surjective representation morphism $T: R_{\Sigma^{\prime}, \mu^{\prime}} \rightarrow R_{\Sigma_{\min }, \mu_{\min }}$. By Lemma 3.8 we get that the latter is equivalent to $T:\left(\Sigma^{\prime}, \mu^{\prime}\right) \rightarrow\left(\Sigma_{\min }, \mu_{\min }\right)$ being a surjective bilinear switched system morphism. From [6], Theorem 4.2, it follows that if $\left(\Sigma^{\prime}, \mu^{\prime}\right)$ is a minimal realization of $\Phi$, then there exists a representation isomorphism $T: R_{\Sigma^{\prime}, \mu^{\prime}} \rightarrow R_{\Sigma_{\min }, \mu_{\min }}$ which means that $\left(\Sigma_{\min }, \mu_{\min }\right)$ gives rise to the bilinear switched system isomorphism $T:\left(\Sigma^{\prime}, \mu^{\prime}\right) \rightarrow\left(\Sigma_{\min }, \mu_{\min }\right)$, that is, $\left(\Sigma^{\prime}, \mu^{\prime}\right)$ and $\left(\Sigma_{\min }, \mu_{\min }\right)$ are isomorphic.

In the procedures below we assume that $\Phi$ is a family of input-output maps with arbitrary switching.

Procedure 3.1 (construction of a minimal realization from the Hankel-matrix). Using [6], Procedure B.1, construct a minimal representation $R$ of $\Psi_{\Phi}$ from the Hankel-matrix $H_{\Phi}=H_{\Psi_{\Phi}}$. Construct the bilinear switched system realization $\left(\Sigma_{R}, \mu_{R}\right)$ associated with $R$. By Lemma $3.7,\left(\Sigma_{R}, \mu_{R}\right)$ is a minimal realization of $\Phi$.

Procedure 3.2 (minimization). Let $(\Sigma, \mu)$ be a bilinear switched system realization of $\Phi$ and compute the associated rational representation $R_{\Sigma, \mu}$ of $\Psi_{\Phi}$. Use [6], Procedure B.4, to transform $R_{\Sigma, \mu}$ into a minimal rational representation $R$ of $\Psi_{\Phi}$. Construct the bilinear switched system realization $\left(\Sigma_{R}, \mu_{R}\right)$ associated with $R$. Then by Lemma 3.7, $\left(\Sigma_{R}, \mu_{R}\right)$ will be a minimal bilinear switched system realization of $\Phi$.

\subsection{Proof of the main results: constrained switching}

\subsubsection{Existence of a realization: proof of Theorem 2.7}

For the rest of the section, let $L \subseteq Q^{+}$be the set of admissible sequences of discrete modes and let $\Phi$ be a family of input-output maps with the switching constraint $L$. We also assume that $\Phi$ admits a generalized Fliess-series expansion. We will follow the same approach as in Section 3.1.1 for the case of arbitrary switching. That is, we associate with $\Phi$ a family of formal power series $\Psi_{\Phi}$ and we will relate realizations of $\Phi$ with representations of $\Psi_{\Phi}$. The family $\Psi_{\Phi}$ will consist of formal power series indexed by elements $\Phi$. An element $T_{f}$ of $\Psi_{\Phi}$ indexed by $f \in \Phi$ will contain an encoding of the values of the GCGS $c_{f}$ which generates $f$. For those sequences, for which $c_{f}$ is not defined, the value of $T_{f}$ will be zero.

Below we formalize the approach outlined above. To this end, recall from (2.25), Section 2.4 the definition of the vectors $T_{f, q}(s)$ for $f$ of $\Phi, q \in Q$ and $s \in \Gamma^{*}$. Define that formal power series $T_{f, q}$ as $T_{f, q}: \Gamma^{*} \ni s \mapsto$ $T_{f, q}(s) \in \mathbb{R}^{p}$. Below we will formally prove that $T_{f, q}(s)$ is indeed well-defined for any word $s \in \Gamma^{*}$.

Lemma 3.9. For any $s \in \Gamma^{*}$ the value of $T_{f, q}(s)$ as defined in $(2.4)$ is uniquely defined. Hence, the function $T_{f, q}$ is a well-defined formal power series.

The proof of Lemma 3.9 is based on the possibility of extending any GCGS $c$ defined on $J L$ to a GCGS defined on $\widetilde{J L}$ ( see $(2.24)$ for the definition of $\widetilde{J L}$ ). The existence of such an extension not only provides a proof of Lemma 3.9, but it is also useful for simplifying notation in the subsequent proofs. The definition goes as follows.

Definition 3.1. Consider a GCGS $c: J L \rightarrow \mathcal{Y}$ and the set $\widetilde{J L}$ from (2.24). Define the GCGS $\widetilde{c}: \widetilde{J L} \rightarrow \mathcal{Y}$ as follows; for any $s \in \widetilde{J L}$ choose a word $w \in J L$ such that $(s, w) \in R^{*}$ and let $\widetilde{c}(s)=c(w)$.

Lemma 3.10. This extension $\widetilde{c}$ is well-defined. In addition, $c$ and $\widetilde{c}$ coincide on $J L$.

The proof of Lemma 3.10 can be found in Appendix A. Now we are ready to present the proof of Lemma 3.9. 
Proof of Lemma 3.9. Let us apply Definition 3.1 to the GCGS $c_{f}$ and denote the resulting extension of $c_{f}$ by $\widetilde{c}_{f}$. Notice that (2.25) implies that $T_{f, q}(s)$ is of the form $T_{f, q}(s)=\left\{\begin{array}{cc}\widetilde{c}_{f}(s(q, \epsilon)) & \text { if } s(q, \epsilon) \in \widetilde{J L} \\ 0 & \text { otherwise }\end{array}\right.$. Hence, the value of $T_{f, q}(s)$ is uniquely determined for all $s \in \Gamma^{*}$.

Notation 3.1. In the sequel, we identify any CGCS $c: J L \rightarrow \mathcal{Y}$ with its extension $\widetilde{c}$ to $\widetilde{J L}$ from Definition 3.1.

Recall the enumeration $Q=\left\{\sigma_{1}, \sigma_{2}, \ldots, \sigma_{N}\right\}$ of the discrete modes defined in (2.23). Define the formal power series $T_{f} \in \mathbb{R}^{N p} \ll \Gamma^{*} \gg$ by requiring that for all $s \in \Gamma^{*}$,

$$
T_{f}(s)=\left[\begin{array}{llll}
\left(T_{f, \sigma_{1}}(s)\right)^{T} & \left(T_{f, \sigma_{2}}(s)\right)^{T} \quad \ldots \quad\left(T_{f, \sigma_{N}}(s)\right)^{T}
\end{array}\right]^{T} .
$$

That is, the values of $T_{f}$ are formed by vertically "stacking up" the values of $T_{f, \sigma_{1}}, T_{f, \sigma_{2}}, \ldots, T_{f, \sigma_{N}}$, in this order, starting from the top and ending in the bottom. The intuition behind the definition of $T_{f}$ is the following. The values of $T_{f}$ are just simply encodings of the values the GCGS $c_{f}$ whenever $c_{f}$ is well-defined and zero otherwise. Recall the formal power series $S_{f}$ from (3.1) defined for the case of arbitrary switching. The series $T_{f}$ is completely analogous $S_{f}$; the only difference is that we replace $S_{f, \sigma_{i}}$ by $T_{f, \sigma_{i}}$ for all $i=1, \ldots, N$. Notice that if $c_{f}$ is defined for any sequence, then the definition of $S_{f, \sigma_{i}}$ and $T_{f, \sigma_{i}}$ coincide, and hence in this case $S_{f}$ and $T_{f}$ coincide as well. Define the set of formal power series $\Psi_{\Phi}$ associated with $\Phi$ as follows

$$
\Psi_{\Phi}=\left\{T_{f} \in \mathbb{R}^{N p} \ll \Gamma^{*} \gg \mid f \in \Phi\right\} .
$$

That is, $\Psi_{\Phi}$ is simply the collection of the formal power series $T_{f}$.

Remark 3.2 (equivalence of Hankel-matrix definitions). The Hankel-matrix $H_{\Phi}$ of $\Phi$ as defined in Definition 2.9 and the Hankel-matrix $H_{\Phi_{\Psi}}$ of $\Phi_{\Psi}$ coincide, and hence their ranks coincide too.

In order to prove Theorem 2.7 we first state a number of results, proof of which will be presented at the end of the section. Recall from [6], equation (4.1), the notion of Hadamard product.

Theorem 3.2. There exists a family of formal power series $\Omega_{\Phi}$ such that the following holds. If $(\Sigma, \mu)$ is a bilinear switched system realization of $\Phi$, then there exists a family of formal power series $K_{\Sigma, \mu}$ such that

(i) The family $K_{\Sigma, \mu}$ is rational and its elements depend only on the parameters of $(\Sigma, \mu)$.

(ii) The family of formal power series $\Psi_{\Phi}$ is the Hadamard product of $K_{\Sigma, \mu}$ and $\Omega_{\Phi}$, i.e.

$$
\Psi_{\Phi}=K_{\Sigma, \mu} \odot \Omega_{\Phi}
$$

Moreover, the elements of $\Omega_{\Phi}$ depend only on $L$, and if $L$ is regular, then $\Omega_{\Phi}$ is rational.

The proof of the theorem will be presented later. The theorem above has the following simple corollary.

Corollary 3.1. If $\Phi$ has a bilinear switched system realization and $L$ is a regular language, then $\Psi_{\Phi}$ is rational.

Proof of Corollary 3.1. Take a bilinear switched system realization $(\Sigma, \mu)$ of $\Phi$. From Theorem 3.2 we get that $K_{\Sigma, \mu}$ is rational, and if $L$ is regular, then $\Omega_{\Phi}$ is rational too. From [6], Lemma 4.3, it follows that the Hadamard product of two rational families is rational, and hence from (3.12) we get that $\Psi_{\Phi}$ is rational.

In the sequel we will state the converse of Corollary 3.1. That is, we will claim that if $\Psi_{\Phi}$ is rational, then $\Phi$ has a bilinear switched system realization with constraint $L$. To this end, recall from Construction 3.2 the definition of a bilinear switched system realization associated with a representation. Recall from [6], equation (3.7), the definition of $\operatorname{comp}(L)$.

Theorem 3.3. If $R=\left(\mathcal{X},\left\{B_{\sigma}\right\}_{\sigma \in \Gamma}, I, \widetilde{C}\right)$ is a representation of $\Psi_{\Phi}$, then $\left(\Sigma_{R}, \mu_{R}\right)$ is a realization of $\Phi$. Moreover, for each $f \in \Phi, w \in T(\operatorname{comp}(L))$, and $u \in P C(T, \mathcal{U}), y_{\Sigma_{R}}\left(\mu_{R}(f), u, w\right)=0$. 
The proof of the theorem will be presented at the end of the section.

Proof of Theorem 2.7. Recall from Remark 3.2 that the Hankel-matrix of $H_{\Psi_{\Phi}}$ and the Hankel-matrix of $\Phi$ as defined in Construction 2.9 coincide. Hence, if the Hankel-matrix $H_{\Phi}$ is of finite rank, then it means that the Hankel-matrix $H_{\Psi_{\Phi}}$ of $\Psi_{\Phi}$ is of finite rank. Hence, by [6], Theorem 4.1, $\Psi_{\Phi}$ is rational. Take a representation $R$ of $\Psi_{\Phi}$ and apply Theorem 3.3 to $R$. We get that $\left(\Sigma_{R}, \mu_{R}\right)$ is a realization of $\Phi$. That is, first statement of the theorem is proved. Assume that $L$ is regular and $\Phi$ has a realization by a bilinear switched system. Then Corollary 3.1 implies that $\Psi_{\Phi}$ is rational. By [6], Theorem 4.1, the latter implies that $H_{\Psi_{\Phi}}=H_{\Phi}$ is of finite rank.

We will proceed with the proof of Theorem 3.2. We start with defining the family of formal power series $\Omega_{\Phi}$ from Theorem 3.2. Recall from $(2.24)$ the the set $\widetilde{J L} \subseteq \widetilde{\Gamma}^{*}$. For each discrete mode $q \in Q$ define the set

$$
L_{q}=\left\{s \in \Gamma^{*} \mid s(q, \epsilon) \in \widetilde{J L}\right\}
$$

Lemma 3.11. $s \in L_{q}$ if and only if there exist $w \in \widetilde{\Gamma}^{*},(q, v) \in \widetilde{\Gamma}$ such that $w(q, v) \in J L$ and $(s(q, \epsilon)$, $w(q, v)) \in R^{*}$.

Lemma 3.12. If $L$ is regular, then the languages $L_{q}, q \in Q$ are regular.

The proof of Lemmas 3.11 and 3.12 can be found in Appendix A. Lemma 3.11 implies that for any $f \in \Phi$, and any word $s \in L_{q}, c_{f}(s(q, \epsilon))$ is defined. For each $q \in Q$ and $s \in \Gamma^{*}$, define $Z_{q}(s) \in \mathbb{R}^{p}$ as

$$
Z_{q}(s)=\left\{\begin{aligned}
(1,1, \ldots, 1)^{T} & \text { if } s \in L_{q} \\
0 & \text { otherwise. }
\end{aligned}\right.
$$

Recall from Section 2.4, (2.23) the enumeration $Q=\left\{\sigma_{1}, \ldots, \sigma_{N}\right\}$. Define the formal power series $Z \in \mathbb{R}^{N p}$ $\ll \Gamma \gg$ by requiring that for each word $s \in \Gamma^{*}$,

$$
Z(s)=\left[\begin{array}{llll}
\left(Z_{\sigma_{1}}(s)\right)^{T} & \left(Z_{\sigma_{2}}(s)\right)^{T} \quad \ldots \quad\left(Z_{\sigma_{N}}(s)\right)^{T}
\end{array}\right]^{T} .
$$

That is, $Z(s)$ is obtained by "stacking up" the values of $Z_{\sigma_{1}}(s), Z_{\sigma_{2}}(s), \ldots, Z_{\sigma_{N}}(s)$ in this order, from top to bottom. We define the family of formal power series $\Omega_{\Phi}$ as follows:

$$
\Omega_{\Phi}=\left\{Z_{f} \in \mathbb{R}^{p N} \ll \Gamma^{*} \gg \mid f \in \Phi\right\} \text { where } Z_{f}=Z \text { for all } f \in \Phi .
$$

Corollary 3.2. If $L$ is regular, then $\Omega_{\Phi}$ is rational. In addition, rank $H_{\Omega_{\Phi}}$ depends only on the language $L$.

Proof. First we show the formal power series $Z$ is rational, if $L$ is regular. From this, the statement of the corollary follows. Indeed, assume that $\{Z\}$ is rational and let $R=\left(\mathcal{X},\left\{A_{\sigma}\right\}_{\sigma \in \Gamma},\{B\}, C\right)$ be a minimal, and hence reachable and observable, representation of $\{Z\}$. Then define the indexed set $\widetilde{B}=\left\{\widetilde{B}_{f} \mid \in \Phi\right\}$ by $\widetilde{B}_{f}=B$ for all $f \in \Phi$. It is easy to see that $\widetilde{R}=\left(\mathcal{X},\left\{A_{\sigma}\right\}_{\sigma \in \Gamma}, \widetilde{B}, C\right)$ is a representation of $\Omega_{\Phi}$. Hence, $\Omega_{\Phi}$ is indeed rational. It is easy to see that $\widetilde{R}$ is reachable and observable, and hence minimal. Hence, $\operatorname{rank} H_{\Omega_{\Phi}}=\operatorname{dim} R=\operatorname{dim} \widetilde{R}=\operatorname{rank} H_{\{Z\}}$. Notice that the definition of $Z$ depends only on $L$, hence, the rank of the Hankel matrix of $Z$ depends only on $L$ as well.

Hence, it is left to show that $Z$ is rational. Notice that for any $l=1,2, \ldots, N p, l$ is always of the form $l=p(z-1)+i$ for some $z=1,2, \ldots, N$ and $i=1,2, \ldots, p$. From (3.14) it follows that for all $s \in \widetilde{\Gamma}^{*}$, the $l$ th coordinate of $Z(s)$ is of the form $(Z(s))_{l}=\left\{\begin{array}{ll}1 & \text { if } s \in L_{\sigma_{z}} \\ 0 & \text { otherwise }\end{array}\right.$. Consider the formal power series $Z_{l}: \widetilde{\Gamma}^{*} \ni s \mapsto$ $(Z(s))_{l} \in \mathbb{R}$. By [6], Lemma 4.2, the regularity of $L_{\sigma_{l}}$ implies that $Z_{l}$ is rational for all $l=1, \ldots, N p$. From [6], Lemma 4.4 , it follows then that $\{Z\}$ is rational. 
Consider a bilinear switched system realization $(\Sigma, \mu)$, where $\Sigma$ is of the form (2.1) and $\mu: \Phi \rightarrow \mathcal{X}$. As the next step, we will define the family of formal power $K_{\Sigma, \mu}$ from Theorem 3.2. Recall from [6], Section 2, the definition of the input-output map $y_{\Sigma}(\mu(f), . .$.$) of \Sigma$ induced by the state $\mu(f)$. Consider the family of input-output maps $\Theta_{\Sigma, \mu}=\left\{y_{\Sigma}(\mu(f), . .) \mid. f \in \Phi\right\}$ defined for arbitrary switching. The following holds for $\Theta_{\Sigma, \mu}$.

Lemma 3.13. $\Sigma$ is a realization of $\Theta_{\Sigma, \mu}$. Hence, $\Theta_{\Sigma, \mu}$ has a generalized Fliess-series expansion and the family of formal power series $\Psi_{\Theta_{\Sigma, \mu}}$ associated with $\Theta_{\Sigma, \mu}$, as defined in Section 3.1.1, (3.2), is rational.

Proof. Consider the map $\widetilde{\mu}: \Theta_{\Sigma, \mu} \rightarrow \mathcal{X}$ such that for each $g \in \Theta_{\Sigma, \mu} \widetilde{\mu}(g)=\mu(f)$ where $f$ is an element $\Phi$ such that $g=y_{\Sigma}(\mu(f), . .$.$) . Assume that there is another f^{\prime} \in \Phi$, such that $g=y_{\Sigma}\left(\mu\left(f^{\prime}\right), . ..\right)$. But $(\Sigma, \mu)$ is a realization of $\Phi$, hence it follows that for all $u \in P C(T, \mathcal{U}), w \in T L, f(u, w)=g(u, w)=f^{\prime}(u, w)$, i.e. $f=f^{\prime}$. Hence, $\widetilde{\mu}$ is well-defined. Then it follows that $y_{\Sigma}(\widetilde{\mu}(g), .,)=$.$g for all g \in \Theta_{\Sigma, \mu}$, hence $(\Sigma, \widetilde{\mu})$ is a realization of $\Theta_{\Sigma, \mu}$. The rest of the lemma follows from Theorem 3.1.

Now we are ready to define the family of formal power series $K_{\Sigma, \mu}$. Assume that

$$
\Psi_{\Theta_{\Sigma, \mu}}=\left\{S_{g} \in \mathbb{R}^{N p} \ll \Gamma^{*} \gg \mid g \in \Theta_{\Sigma, \mu}\right\} .
$$

For each map $f \in \Phi$, denote by $G_{f}$ the formal power series $S_{g}$ where $g=y_{\Sigma}(\mu(f), . .$.$) . Then define K_{\Sigma, \mu}$ as

$$
K_{\Sigma, \mu}=\left\{G_{f} \in \mathbb{R}^{p} \ll \Gamma^{*} \gg \mid f \in \Phi\right\} .
$$

That is, $K_{\Sigma, \mu}$ is simply a re-indexing of $\Psi_{\Theta_{\Sigma, \mu}}$. The reason this re-indexing is necessary is that $\Psi_{\Phi}$ is indexed by $\Phi$, hence both families on the right-hand side of (3.12) have to be indexed by $\Phi$. We have the following.

Lemma 3.14. The family $K_{\Sigma, \mu}$ is rational and rank $H_{K_{\Sigma, \mu}}=\operatorname{rank} H_{\Theta_{\Sigma, \mu}}$.

Proof. Define the map $U: \Phi \rightarrow \Theta_{\Sigma, \mu}$ by $U(f)=y_{\Sigma}(\mu(f), . .$.$) . It is easy to see that G_{f}=S_{U(f)}$ and $U$ is surjective, and from Lemma 3.13 it follows that $\Psi_{\Theta_{\Sigma, \mu}}$ is rational. Hence, from [6], Lemma 4.5, we get that $K_{\Sigma, \mu}$ is rational and rank $H_{K_{\Sigma, \mu}}=\operatorname{rank} H_{\Psi_{\Theta_{\Sigma, \mu}}}=\operatorname{rank} H_{\Theta_{\Sigma, \mu}}$.

Proof of Theorem 3.2. Part (i) of the theorem follows easily from the construction of $K_{\Sigma, \mu}$ and Lemma 3.14. Next, we will prove Part (ii) of the theorem. Recall from the proof of Lemma 3.13 the map $\widetilde{\mu}: \Theta_{\Sigma, \mu} \rightarrow \mathcal{X}$. For each $f \in \Phi$, denote by $c_{f}: \widetilde{J L} \rightarrow \mathcal{Y}$ the GCGS which generates $f$, i.e. $F_{c_{f}}=f$. Denote by $d_{f}: \widetilde{\Gamma}^{*} \rightarrow \mathcal{Y}$ the GCGS which generates $y_{\Sigma}(\mu(f), .,$.$) , i.e. F_{d_{f}}=y_{\Sigma}(\mu(f), .,$.$) .$

Recall the equality (3.5). Using this equality and Theorem 2.5, we get that $(\Sigma, \mu)$ is a realization of $\Phi$ with constraint $L$, if and only if for all $f \in \Phi, w \in \widetilde{\Gamma}^{*}$ and $(q, v) \in \widetilde{\Gamma}$ such that $w(q, v) \in J L$,

$$
c_{f}(w(q, v))=C_{q} B_{q, v} B_{\phi(w)} \mu(f) .
$$

Similarly, by applying (3.5) and using Theorem 2.5 for the case of $\Theta_{\Sigma, \mu}$, we get that $(\Sigma, \widetilde{\mu})$ is a realization of $\Theta_{\Sigma, \mu}$ if and only if for each $f \in \Phi$, and for each $w \in \widetilde{\Gamma}^{*}$ and $(q, v) \in \widetilde{\Gamma}$

$$
d_{f}(w(q, v))=C_{q} B_{q, v} B_{\phi(w)} \widetilde{\mu}\left(y_{\Sigma}(\mu(f), . . .)\right) .
$$

Recall from (3.13) the definition of the set $L_{q}$. By Lemma 3.11, if $s \in L_{q}$, then there exists a word $w(q, v) \in J L$ such that $(w(q, v), s(q, \epsilon)) \in R^{*}$. Hence, if $s \in L_{q}$, then $c_{f}(s(q, \epsilon))=c_{f}(w(q, v))$, and $d_{f}(s(q, \epsilon))=d_{f}(w(q, v))$ holds. By applying the equality above, (3.18) and (3.19) to $w(q, v)$, and using $\mu(f)=\widetilde{\mu}\left(y_{\Sigma}(\mu(f), . .).\right)$, we get $c_{f}(s(q, \epsilon))=C_{q} B_{q, v} B_{\phi(w)} \mu(f)=d_{f}(s(q, \epsilon))$. That is, for each $q \in Q, f \in \Phi, s \in L_{q}$ we get that

$$
T_{f, q}(s)=c_{f}(s(q, \epsilon))=d_{f}(s(q, \epsilon))=S_{y_{\Sigma}(\mu(f), ., .), q}(s) .
$$

Notice from (2.25) and (3.14) that for each $s \notin L_{q}, T_{f, q}(s)=0$ and $Z_{q}(s)=0$. That is, $T_{f, q}=S_{f, q} \odot Z_{q}$ and therefore $T_{f}=S_{y_{\Sigma}(\mu(f), . . .)} \odot Z_{f}$, for all $f \in \Phi$. Formula (3.12) follows now from the definition of $K_{\Sigma, \mu}$. 
Proof of Theorem 3.3. First we show that $\left(\Sigma_{R}, \mu_{R}\right)$ is a realization of $\Phi$. Let $(\Sigma, \mu)=\left(\Sigma_{R}, \mu_{R}\right)$ be the realization associated with $R$ as in Construction 3.2 and assume that $\Sigma=\Sigma_{R}$ is of the form (2.1). Recall that by possibly replacing $R$ with an isomorphic copy whose state-space is $\mathbb{R}^{n}, n=\operatorname{dim} R$, we can assume that $R=R_{\Sigma, \mu}$, and hence we can apply (3.5). Using the fact that $R$ is a representation of $\Psi_{\Phi}$, and the construction $\widetilde{C}$ of the readout map of $R$, we get that for all $f \in \Phi$ and $s \in \Gamma^{*}, T_{f, q}(s)=C_{q} B_{s} I_{f}$. Consider a word $v=$ $\left(q_{1}, w_{1}\right)\left(q_{2}, w_{2}\right) \ldots\left(q_{k}, w_{k}\right) \in J L, q_{1}, \ldots, q_{k} \in Q, w_{1}, \ldots, w_{k} \in \mathrm{Z}_{m}^{*}, k>0$. From Lemma 3.3 it follows that $\left(v\left(q_{k}, \epsilon\right), \phi(v)\left(q_{k}, \epsilon\right)\right) \in R^{*}$ and evidently $\left(v\left(q_{k}, \epsilon\right), v\right) \in R^{*}$. Hence, $\left(\phi(v)\left(q_{k}, \epsilon\right), v\right) \in R^{*}$ and from (2.25) for all $f \in \Phi, c_{f}(v)=c_{f}\left(\phi(v)\left(q_{k}, \epsilon\right)\right)$. From this, Lemma 3.10, and (3.5) we get that

$$
c_{f}(v)=T_{f, q_{k}}(\phi(v))=C_{q_{k}} B_{\phi(v)} I_{f}=C_{q_{k}} B_{q_{k}, w_{k}} \ldots B_{q_{1}, w_{1}} \mu(f) .
$$

From Theorem 2.5 we get that (3.21) implies that $\left(\Sigma_{R}, \mu_{R}\right)$ is a realization of $\Phi$.

Consider the switching sequence $v=\left(q_{1}, t_{1}\right)\left(q_{2}, t_{2}\right) \ldots\left(q_{k}, t_{k}\right) \in T(\operatorname{comp}(L)), q_{1}, q_{2}, \ldots, q_{k} \in Q, t_{1}, t_{2}, \ldots$, $t_{k} \in T$. We show that for all $u \in P C(T, \mathcal{U}), y_{\Sigma}(\mu(f), u, v)=0$. It follows from [6], equation (3.7), that $\widetilde{L}_{q_{k}}=\emptyset$, where $\widetilde{L}_{q_{k}}$ was defined in [6], equation (5.11). Then for each $s \in \widetilde{\Gamma}^{*}$ of the form $s=\left(q_{1}, w_{1}\right)\left(q_{2}, w_{2}\right) \ldots\left(q_{k}, w_{k}\right)$ for some $w_{1}, w_{2}, \ldots, w_{k} \in \mathrm{Z}_{m}^{*}$, we get that $\phi(s) \notin L_{q_{k}}$. Indeed, $\widetilde{L}_{q_{k}}=\emptyset$ and from the proof of Lemma 3.12, presented in Appendix A, we know that $L_{q}=\operatorname{pr}_{Q}^{-1}\left(\widetilde{L}_{q}\right)$. If $\phi(s) \in L_{q_{k}}$, then we get that $\operatorname{pr}_{Q}(\phi(s)) \in \widetilde{L}_{q_{k}}=\emptyset$, a contradiction. Hence, it follows from the definition $(2.25)$ of $T_{f, q_{k}}$ that $T_{f, q_{k}}(\phi(s))=0$ for each $f \in \Phi$ and $s=\left(q_{1}, w_{1}\right) \ldots\left(q_{k}, w_{k}\right)$, where $q_{1}, \ldots q_{k} \in \operatorname{comp}(L)$. But $g=y_{\Sigma}(\mu(f), . .$.$) has a generalized Fliess-series$ expansion, since it is realized by $\Sigma$. Denote by $c_{g}$ the GCGS which generates $g$, i.e. $c_{g}$ is such that $F_{c_{g}}=g$. Then from Theorem 2.5 and the fact that $R$ is a representation of $\Psi_{\Phi}$, it follows

$$
c_{g}\left(\left(q_{1}, w_{1}\right)\left(q_{2}, w_{2}\right) \ldots\left(q_{k}, w_{k}\right)\right)=C_{q_{k}} B_{q_{k}, w_{k}} B_{q_{k-1}, w_{k-1}} \ldots B_{q_{1}, w_{1}} \mu(f)=C_{q_{k}} B_{\phi(s)} I_{f}=T_{f, q_{k}}(\phi(s))=0 .
$$

Here, we used (3.5) again. Then (3.22) together with the definition of $g=F_{c_{g}}$ implies that $g(u, v)=F_{c_{g}}(u, v)=0$ for $v=\left(q_{1}, t_{1}\right) \ldots\left(q_{k}, t_{k}\right)$ and $u \in P C(T, \mathcal{U})$. Since $g(u, v)=y_{\Sigma}(\mu(f), u, v)$, we get that $y_{\Sigma}(\mu(f), u, v)=0$.

\subsubsection{Quasi-minimality: proof of Theorem 2.4}

Proof of Theorem 2.4. Assume that $L$ is regular and that $\Phi$ has a realization by a bilinear switched system. Then Theorem 2.7 implies that $\Psi_{\Phi}$ is rational. Consider a minimal representation $R$ of $\Psi_{\Phi}$. Construct the bilinear switched system realization $\left(\Sigma_{R}, \mu_{R}\right)$ associated with $R$ as described in Construction 3.2. We claim that $\left(\Sigma_{m}, \mu_{m}\right)=\left(\Sigma_{R}, \mu_{R}\right)$ satisfies the statement of the theorem. Indeed, Theorem 3.3 implies that $\left(\Sigma_{m}, \mu_{m}\right)$ is a realization of $\Phi$ and $(2.16)$ holds for $\left(\Sigma_{m}, \mu_{m}\right)$. Moreover, since $R$ is minimal, it is reachable and observable. Lemma 3.6 then implies that $\left(\Sigma_{m}, \mu_{m}\right)$ is semi-reachable and observable.

It is left to show that $(2.17)$ holds for all realizations $(\Sigma, \mu)$ of $\Phi$. Consider a bilinear switched system realization $(\Sigma, \mu)$ of $\Phi$. Recall from (3.17) the definition of $K_{\Sigma, \mu}$ and recall the definition of the family of input-output map $\Theta_{\Sigma, \mu}$. Notice $K_{\Sigma, \mu}$ is obtained from $\Psi_{\Theta_{\Sigma, \mu}}$ by re-indexing its elements. Recall from the proof of Lemma 3.13 that $(\Sigma, \widetilde{\mu})$, is a realization of $\Theta_{\Sigma, \mu}$. Hence, Theorem 3.1 implies that $R_{\Sigma, \widetilde{\mu}}$ is a representation of $\Psi_{\Theta_{\Sigma, \mu}}$ and hence, the rank of the Hankel-matrix of $\Psi_{\Theta_{\Sigma, \mu}}$ satisfies rank $H_{\Psi_{\Theta_{\Sigma}, \mu}} \leq \operatorname{dim} R_{\Sigma, \tilde{\mu}}=\operatorname{dim} \Sigma$. Since $K_{\Sigma, \mu}$ is just a re-indexing of $\Psi_{\Theta_{\Sigma, \mu}}$, from [6], Lemma 4.5, it follows that rank $H_{K_{\Sigma, \mu}} \leq \operatorname{rank} H_{\Psi_{\Theta_{\Sigma, \mu}}} \leq \operatorname{dim} \Sigma$. Since (3.12) holds for $K_{\Sigma, \mu}$ and $\Psi_{\Phi}$, from [6], Lemma 4.3, and the discussion above it follows that rank $H_{\Psi_{\Phi}}=$ rank $H_{K_{\Sigma, \mu} \odot \Omega_{\Phi}} \leq \operatorname{rank} H_{K_{\Sigma, \mu}} \cdot \operatorname{rank} H_{\Omega_{\Phi}} \leq \operatorname{rank} H_{\Omega_{\Phi}} \cdot \operatorname{dim} \Sigma$. We proceed to prove that (2.17) holds. From [6], Theorem 4.2, it follows that $\operatorname{dim} \Sigma_{m}=\operatorname{dim} R=\operatorname{rank} H_{\Psi_{\Phi}}$. Choosing $M=\operatorname{rank} H_{\Omega_{\Phi}}$ and using the discussion above we get (2.17). The statement that $M$ depends only on $L$ follows from Corollary 3.2.

Procedure 3.3 (construction of a realization from the Hankel-matrix). Assume that $L$ is a regular language. Construct a minimal representation $R$ from $H_{\Phi}$ using [6], Procedure 8.1. Construct the bilinear switched system realization $\left(\Sigma_{R}, \mu_{R}\right)$ associated with $R$. By Theorem $3.3\left(\Sigma_{R}, \mu_{R}\right)$ is a realization of $\Phi$, and from the proof of Theorem 2.4 it follows $\left(\Sigma_{R}, \mu_{R}\right)$ is a quasi-minimal realization of $\Phi$. 
Procedure 3.4 (quasi-minimization). We can transform a bilinear switched system realization $(\Sigma, \mu)$ of $\Phi$ to a quasi-minimal one as follows. Using $(\Sigma, \mu)$ we can construct the Hankel-matrix $H_{\Phi}$ of $\Phi$. Then we can use Procedure 3.3 to construct a a quasi-minimal realization of $\Phi$.

\section{Conclusions}

This paper represents Part II of a series of papers on realization theory of linear and bilinear switched systems. In this paper we presented a solution to the realization problem for bilinear switched systems. The paper uses the theory of formal power series to deal with realization problem for switched systems. Topics of further research include realization theory for piecewise-affine systems, and non-linear switched systems.

Acknowledgements. The author thanks Jan H. van Schuppen, Pieter Collins and Luc Habets for for the useful discussions and suggestions. The author thanks the anonymous referee for the useful comments.

\section{A. TECHNicAl PROOFS}

In Section A.1 a number of results is presented on GCGSs and input-output maps generated by them. Section A.2 presents the proof of Theorems 2.1, 2.5 and 2.2. Section A.3 presents the proof of some technical results used in Sections 3.1 and 3.2.

\section{A.1. Technical results on generalized Fliess-series expansion}

The goal of this section is to present a number of results on input-output maps of bilinear switched systems. In the sequel, we use the notation of Section 2.4. Recall the notion of a GCGS. First, we show that the map $F_{c}$ generated by a GCGS $c$ is well-defined.

Lemma A.1. If $c: J L \rightarrow \mathcal{Y}$ is a GCGS then for each input $u \in P C(T, \mathcal{U})$, and switching sequence $s=$ $\left(q_{1}, t_{1}\right)\left(q_{2}, t_{2}\right) \ldots\left(q_{k}, t_{k}\right) \in T L, q_{1}, \ldots, q_{k} \in Q, t_{1}, \ldots, t_{k} \in T, k>0$, the series $F_{c}(u, s)$ defined in $(2.20)$ is absolutely convergent.

Proof of Lemma A.1. For any natural number $N \in \mathbb{N}$ denote by $W_{N, k}$ the set of all sequences of words $w_{1}, \ldots, w_{k} \in \mathrm{Z}_{m}$, such that $\left|w_{1}\right|+\left|w_{2}\right|+\ldots+\left|w_{k}\right| \leq N$. Define the finite sum $S_{N}$ by

$$
S_{N}=\sum_{w_{1}, w_{2}, \ldots, w_{k} \in W_{N, k}} \| c\left(\left(q_{1}, w_{1}\right)\left(q_{2}, w_{2}\right) \ldots\left(q_{k}, w_{k}\right)\right)|| V_{w_{1}, w_{2}, \ldots, w_{k}}[u]\left(t_{1}, \ldots, t_{k}\right) \mid
$$

In order to show that $F_{c}(u, s)$ is absolutely convergent, we have to show that $S_{N}, N \in \mathbb{N}$ is bounded from above. Since $u$ is piecewise-continuous, there exists $R>1$ such that $\max \left\{\left|u_{j}(t)\right| \mid j=1,2, \ldots, m, t \in\left[0, \sum_{i=1}^{k} t_{i}\right]\right\}<R$. Then by induction it is easy to see that for all $w \in \mathrm{Z}_{m}^{*}$, the absolute value $\left|V_{w}[u]\left(t_{i}\right)\right|$ is bounded from above by $\frac{\left.R^{|w|}\right|_{t} ^{|w|}}{|w| !}$ for all $w \in \mathrm{Z}_{m}^{*}$. Hence, for any sequence $w_{1}, \ldots, w_{k} \in \mathrm{Z}_{m}^{*}$,

$$
\left|V_{w_{1}, w_{2}, \ldots, w_{k}}[u]\left(t_{1}, \ldots, t_{k}\right)\right|=\Pi_{i=1}^{k}\left|V_{w_{i}}[u]\left(t_{i}\right)\right| \leq \frac{t_{1}^{\left|w_{1}\right|}}{\left|w_{1}\right| !} \frac{t_{2}^{\left|w_{2}\right|}}{\left|w_{2}\right| !} \cdots \frac{t_{k}^{\left|w_{k}\right|}}{\left|w_{k}\right| !} R^{\left|w_{1}\right|+\ldots+\left|w_{k}\right|}
$$

Using (A.2) and the fact that $\left\|c\left(\left(q_{1}, w_{1}\right)\left(q_{2}, w_{2}\right) \ldots\left(q_{k}, w_{k}\right)\right)\right\| \leq K M^{\left|w_{1}\right|+\left|w_{2}\right|+\ldots+\left|w_{k}\right|}$, we get that

$$
S_{N} \leq \sum_{w_{1}, \ldots, w_{k} \in W_{N, k}} K(M R)^{\left|w_{1}\right|+\left|w_{2}\right|+\ldots+\left|w_{k}\right|} \frac{t_{1}^{\left|w_{1}\right|}}{\left|w_{1}\right| !} \cdots \frac{t_{k}^{\left|w_{k}\right|}}{\left|w_{k}\right| !} .
$$


For each $l_{1}, \ldots, l_{k} \in \mathbb{N}$ there are precisely $(m+1)^{l_{1}+l_{2}+\ldots+l_{k}}$ sequences of words $w_{1}, \ldots, w_{k} \in \mathrm{Z}_{m}^{*}$ such that $\left|w_{i}\right|=l_{i}, i=1, \ldots k$. Hence, if in (A.3) we collect all the $k$ tuples of words $w_{1}, \ldots, w_{k}$ such that each $w_{i}$, $i=1, \ldots, k$ is of the some fixed length $l_{i}$, we get the following

$$
S_{N} \leq \sum_{l_{1}+\ldots+l_{k} \leq N} K(M R(m+1))^{l_{1}+\ldots+l_{k}} \frac{t_{1}^{l_{1}}}{l_{1} !} \frac{t_{2}^{l_{2}}}{l_{2} !} \cdots \frac{t_{k}^{l_{k}}}{l_{k} !} \leq \sum_{l=0}^{N} K(M R k(m+1))^{l} \frac{T^{l}}{l !} \leq K \mathrm{e}^{M R k(m+1) T}
$$

where $T=\sum_{1}^{k} t_{i}$. Here we used the equality $\sum_{l_{1}+l_{2}+\ldots+l_{k}=l} \frac{l !}{l_{1} ! l_{2} ! \ldots ! l_{k}}=k$. That is, each $S_{N}$ is bounded from above by $K \mathrm{e}^{M R k(m+1) T}$, and hence the series $F_{c}\left(u,\left(q_{1}, t_{1}\right)\left(q_{2}, t_{2}\right) \ldots\left(q_{k}, t_{k}\right)\right)$ is absolutely convergent.

It is a natural to ask whether $c$ determines $F_{c}$ uniquely. The following result answers this question.

Lemma A.2. Let $L \subseteq Q^{*}$ and let $d, c: J L \rightarrow \mathcal{Y}$ be two convergent generating series. If $F_{c}=F_{d}$, then $c=d$.

Proof of Lemma A.2. It is enough to show that for any $k>0$ and any language $L \subseteq Q^{+}$, and any convergent generating series $d, c: J L \rightarrow \mathcal{Y}$, if $F_{d}=F_{c}$ then for each sequence $q_{1} q_{2} \ldots q_{k} \in L, q_{1}, \ldots, q_{k} \in Q$, and each $w_{1}, \ldots, w_{k} \in \mathrm{Z}_{m}^{*}$

$$
c\left(\left(q_{1}, w_{1}\right)\left(q_{2}, w_{2}\right) \ldots\left(q_{k}, w_{k}\right)\right)=d\left(\left(q_{1}, w_{1}\right)\left(q_{2}, w_{2}\right) \ldots\left(q_{1}, w_{k}\right)\right) .
$$

We proceed by induction on $k$. If $k=1$ and $q_{1} \in L$, then consider the series $\widetilde{c}: \mathrm{Z}_{m}^{*} \ni w \mapsto c\left(\left(q_{1}, w\right)\right)$ and $\widetilde{d}: \mathrm{Z}_{m}^{*} \ni w \mapsto d\left(\left(q_{1}, w\right)\right)$. The series $\widetilde{c}$ and $\widetilde{d}$ are convergent series in the sense of [2]. If $F_{c}=F_{d}$, then with the notation of $[8], F_{\widetilde{c}}[u](t)=F_{c}\left(u,\left(q_{1}, t\right)\right)=F_{d}\left(u,\left(q_{1}, t\right)\right)=F_{\widetilde{d}}[u](t)$, which by [2] implies that $\widetilde{c}=\tilde{d}$, that is, $c\left(\left(q_{1}, w\right)\right)=d\left(\left(q_{1}, w\right)\right)$ for each $w \in \mathrm{Z}_{m}^{*}$.

Assume that (A.5) holds for each $k \leq N$ and for any language $L \subseteq Q^{+}$. Let $q_{1} q_{2} \ldots q_{N+1} \in L$ for $q_{1}, \ldots, q_{N+1} \in Q$ and let $c, d: J L \rightarrow \mathbb{R}^{p}$ be two GCGS. Consider the language $H_{q_{1}}=\left\{w \in Q^{+} \mid q_{1} w \in L\right\}$. Denote by $J H_{q_{1}}$ the set of all those sequences $\left(\sigma_{1}, w_{1}\right)\left(\sigma_{2}, w_{2}\right) \ldots\left(\sigma_{k}, w_{k}\right)$ such that $\sigma_{1}, \sigma_{2}, \ldots, \sigma_{k} \in Q$, $w_{1}, w_{2}, \ldots, w_{k} \in \mathrm{Z}_{m}^{*}$ and the sequence $\sigma_{1} \sigma_{2} \ldots \sigma_{k} \in H_{q_{1}}$. Let $w \in \mathrm{Z}_{m}^{*}$ and define the $\operatorname{map} c_{\left(q_{1}, w\right)}: J H_{q_{1}} \rightarrow \mathcal{Y}$, as

$$
c_{\left(q_{1}, w\right)}(s)=c\left(\left(q_{1}, w\right) s\right)
$$

It is easy to see that for all $v=\left(s_{1}, z_{1}\right) \ldots\left(s_{k}, z_{k}\right) \in J H_{q_{1}}, s_{1}, \ldots, s_{k} \in Q, z_{1}, \ldots, z_{k} \in \mathrm{Z}_{m}^{*},\left\|c_{\left(q_{1}, w\right)}(v)\right\|<$ $K M^{|w|} K M^{\left|z_{1}\right|+\ldots+\left|z_{k}\right|}$, i.e. $c_{q_{1}, w}$ is a GCGS. Recall from [6], equation (2.5), that by $T H_{q_{1}}$ we denote the set of all the switching sequences $s=\left(\sigma_{1}, \tau_{1}\right)\left(\sigma_{2}, \tau_{2}\right) \ldots\left(\sigma_{k}, \tau_{k}\right) \in(Q \times T)^{*}$ such that $\sigma_{1}, \ldots, \sigma_{k} \in Q$ and $\sigma_{1} \sigma_{2} \ldots \sigma_{k} \in H_{q_{1}}$. Fix a switching sequence $r=\left(\sigma_{1}, \tau_{1}\right) \ldots\left(\sigma_{k}, \tau_{k}\right) \in T H_{q_{1}}, \tau_{1}, \ldots, \tau_{k} \in T$ and fix a input $u \in P C(T, \mathcal{U})$. Since $c_{\left(q_{1}, w\right)}$ is a GCGS, it induces an input-output map $F_{c_{\left(q_{1}, w\right)}}$ with a well-defined value $F_{c_{\left(q_{1}, w\right)}}(u, r)$ at $(u, r)$. Define the map

$$
F_{c, q_{1}}(u, r): \mathrm{Z}_{m}^{*} \ni w \mapsto F_{c_{\left(q_{1}, w\right)}}(u, r) .
$$

We claim that $F_{c, q_{1}}(u, r)$ is a generating convergent series in the classical sense of [2,8]. Indeed, from the proof of Lemma A.1 it follows that $\left\|F_{c_{\left(q_{1}, w\right)}}\left(u,\left(\sigma_{1}, \tau_{1}\right) \ldots\left(\sigma_{k}, \tau_{k}\right)\right)\right\| \leq M^{|w|} K \mathrm{e}^{M R N(m+1) \sum_{i=1}^{k} \tau_{i}}$ for some $K>0$, where $R \geq \max \left\{1, \max \left\{\left|u_{j}(t)\right| \mid j=1,2, \ldots, m, t \in\left[0, \sum_{1}^{l} \tau_{i}\right]\right\}\right\}$. That is, $R, K$ and $\mathrm{e}^{M R N(m+1)\left(\sum_{i=2}^{N+1} t_{i}\right)}$ are independent of $w$ and hence $F_{c q_{1}, w}$ is indeed a convergent generating series in the classical sense. In the next step, we need the notion of concatenation of two input functions; for any pair of inputs $f, g \in P C\left(T, \mathbb{R}^{m}\right)$, and any time instance $\tau \in T$ the concatenation $f \#_{\tau} g \in P C\left(T, \mathbb{R}^{m}\right)$ of $f$ and $g$ at time instance $\tau$ is defined as $f \#_{\tau} g(t)=\left\{\begin{array}{ll}f(t) & \text { if } t \leq \tau \\ g(t) & \text { if } t>\tau\end{array}\right.$. With the notation above, for any $v \in P C(T, \mathcal{U})$

$$
F_{c}\left(v \#_{t_{1}} u,\left(q_{1}, t_{1}\right) r\right)=\sum_{w \in Z_{m}^{*}} F_{c_{q_{1}, w}}(u, r) V_{w}[v]\left(t_{1}\right)
$$


That is, the value of $F_{c}$ for $v \#_{t_{1}} u$ and $\left(q_{1}, t_{1}\right)\left(\sigma_{1}, \tau_{1}\right) \ldots\left(\sigma_{k}, \tau_{k}\right)$ is the same as output of the input-output map induced by the classical convergent generating series $F_{c, q_{1}}$ for $v$. We use this fact in the induction step below.

For the convergent generating series $d$ define the convergent series $d_{q_{1}, w}$ the map $F_{d_{q_{1}, w}}$ and the corresponding convergent generating series $F_{d, q_{1}}(u, r)$ in a similar way as it was done for $c$ in (A.6), and (A.7).

Assume that $F_{c}=F_{d}$ holds. In particular, we get that for all inputs $v \in P C(T, \mathcal{U})$, and for all $t_{1} \in T$,

$$
F_{c}\left(v \#_{t} u,\left(q_{1}, t_{1}\right) r\right)=F_{d}\left(v \#_{t} u,\left(q_{1}, t_{1}\right) r\right) .
$$

By taking into account (A.8), by induction hypothesis for $k=1$ we get that for each fixed $u \in P C(T, \mathcal{U})$, for each switching sequence $r \in T H_{q_{1}}$, and for all $w \in \mathrm{Z}_{m}^{*} F_{c_{q_{1}, w}}(u, r)=F_{d_{q_{1}, w}}(u, r)$. Then by induction hypothesis for $k=N$ and for $L=H_{q_{1}}$ we get that $c_{q_{1}, w}(v)=d_{q_{1}, w}(v)$ for all words $w \in \mathrm{Z}_{m}^{*}$, and $v \in J H_{q_{1}}$ such that $|v| \leq N$. In particular, for each sequence of words $w_{1}, w_{2}, \ldots, w_{N+1} \in \mathrm{Z}_{m}^{*}$, we get

$$
c\left(\left(q_{1}, w_{1}\right)\left(q_{2}, w_{2}\right) \ldots\left(q_{N+1}, w_{N+1}\right)\right)=c_{\left(q_{1}, w_{1}\right)}(x)=d_{\left(q_{1}, w_{1}\right)}(x)=d\left(\left(q_{1}, w_{1}\right)\left(q_{2}, w_{2}\right) \ldots\left(q_{N+1}, w_{N+1}\right)\right)
$$

where $x=\left(q_{2}, w_{2}\right) \ldots\left(q_{N+1}, w_{N+1}\right)$. But (A.10) is the same as (A.5), hence (A.5) holds for $k \leq N+1$.

\section{A.2. Proof of Theorems 2.1, 2.5 and 2.2}

Proof of Theorem 2.1. To show absolute convergence of the series we will use the notion of a convergent generating series defined in Section 2.4.1. Using the notation of Section 2.4.1 define the series $c_{x_{0}}: \widetilde{\Gamma}^{+} \rightarrow \mathcal{X}$ by $c_{x_{0}}\left(\left(q_{1}, w_{1}\right) \ldots\left(q_{k}, w_{k}\right)\right)=B_{q_{k}, w_{k}} \ldots B_{q_{1}, w_{1}} x_{0}$ for all $q_{1}, \ldots, q_{k} \in Q, w_{1}, \ldots, w_{k} \in \mathrm{Z}_{m}^{*}, k>0$. Then $\left\|c_{x_{0}}\right\| \leq\left\|x_{0}\right\| M^{\sum_{i=1}^{k}\left|w_{i}\right|}$, where $M=\max \left\{\left\|B_{q, j}\right\| \mid q \in Q, j \in \mathrm{Z}_{m}\right\}$. That is, $c_{x_{0}}$ is a convergent generating series and by Lemma A.1 the series on the right-hand side of the first equation of (2.11) is absolutely convergent and equals $F_{c_{x_{0}}}(u, s)$. This also implies the absolute convergence of the right-hand side of the second equation of (2.11).

It is left to show that the right-hand sides of (2.11) equal the respective left-hand sides. We will proceed by induction on $k$. If $k=1$, then $x_{\Sigma}\left(x_{0}, u,\left(q_{1}, t\right)\right)$ is the state under input $u$ at time $t$ of the bilinear system $\dot{x}(t)=A_{q_{1}} x(t)+\sum_{j=1}^{m}\left(B_{q_{1}, j} x\right) u_{j}$ with initial state $x_{0}$. By classical results [2] on bilinear systems $x_{\Sigma}\left(x_{0}, u,\left(q_{1}, t\right)\right)=\sum_{w \in Z_{m}^{*}} B_{q, w} x_{0} V_{w}[u](t)$. Assume that the statement of the proposition is true for all $k \leq N$. Notice that for each $s=\left(q_{1}, t_{1}\right)\left(q_{2}, t_{2}\right) \ldots\left(q_{N}, t_{N}\right) \in(Q \times T)^{*}, x_{\Sigma}\left(x_{0}, u, s\left(q_{N+1}, t_{N+1}\right)\right)=$ $x_{\Sigma}\left(x_{\Sigma}\left(x_{0}, u, s\right), \operatorname{Shift}_{\sum_{1}^{N} t_{i}}(u),\left(q_{N+1}, t_{N+1}\right)\right)$. Using the induction hypothesis one gets

$$
\begin{aligned}
& x_{\Sigma}\left(x_{0}, u, s\left(q_{N+1}, t_{N+1}\right)\right)=\sum_{w_{N+1} \in \mathrm{Z}_{m}^{*}} B_{q_{N+1}, w_{N+1}} x_{\Sigma}\left(x_{0}, u, s\right) V_{w_{N+1}}\left[u_{N}\right]\left(t_{N+1}\right) \\
& =\sum_{w_{N+1} \in \mathrm{Z}_{m}^{*}} B_{q_{N+1}, w_{N+1}} V_{w_{N+1}}\left[u_{N}\right]\left(t_{N+1}\right)\left[\sum_{w_{1}, \ldots, w_{N} \in \mathrm{Z}_{m}^{*}} B_{q_{N}, w_{N}} \ldots B_{q_{1}, w_{1}} x_{0} V_{w_{1}, \ldots, w_{N}}[u]\left(t_{1}, \ldots, t_{N}\right)\right] \\
& =\sum_{w_{1}, \ldots, w_{N+1} \in \mathrm{Z}_{m}^{*}} B_{q_{N+1}, w_{N+1}} B_{q_{N}, w_{N}} \ldots B_{q_{1}, w_{1}} x_{0} V_{w_{1}, w_{2}, \ldots, w_{N+1}}[u]\left(t_{1}, \ldots, t_{N+1}\right)
\end{aligned}
$$

where $u_{N}=\operatorname{Shift}_{\sum_{i=1}^{N} t_{i}}(u)$. The rest of the statement of the theorem follows easily from the fact that $y_{\Sigma}\left(x_{0}, u,\left(q_{1}, t_{1}\right)\left(q_{2}, t_{2}\right) \ldots\left(q_{k}, t_{k}\right)\right)=C_{q_{k}} x_{\Sigma}\left(x_{0}, u,\left(q_{1}, t_{1}\right)\left(q_{2}, t_{2}\right) \ldots\left(q_{k}, t_{k}\right)\right)$.

Proof of Theorem 2.5. If $(\Sigma, \mu)$ is a realization of $\Phi$, then by Theorem 2.1, for each $f \in \Phi$, for each $u \in P C(T, \mathcal{U})$, and $w=\left(q_{1}, t_{1}\right) \ldots\left(q_{k}, t_{k}\right) \in T L$, with $q_{1}, q_{2}, \ldots, q_{k} \in Q, t_{1}, t_{2}, \ldots, t_{k} \in T, k>0, f(u, w)=y_{\Sigma}(\mu(f), u, w)$ equals the right-hand side of the second equation of (2.11). But the right-hand side of the second equation of (2.11) evidently equals $F_{c_{f}}(u, w)$, i.e. it equals the value at $(u, w)$ of the input-output map generated by the GCGS $c_{f}$ defined in (2.21). That is, $f(u, w)=F_{c_{f}}(u, w)$, i.e. $\Phi$ admits a generalized Fliess-series expansion of the form given in (2.21). Conversely, if $\Phi$ admits a generalized Fliess-series expansion of the form (2.21), 
then by using the definition of $F_{c_{f}}$, one gets that $f(u, w)=F_{c_{f}}(u, w)$ equals the right-hand side of the second equation of (2.11). From Theorem 2.1 it then follows that $f(u, w)=F_{c_{f}}(u, w)=y_{\Sigma}(\mu(f), u, w)$, i.e. $(\Sigma, \mu)$ is a realization of $\Phi$.

Proof of Theorem 2.2. Part (i). For each $\mathcal{X}_{0} \subseteq \mathcal{X}, q_{1}, q_{2}, \ldots, q_{k} \in Q, k>0$ define the set $W_{q_{1} q_{2} \ldots q_{k}}\left(\mathcal{X}_{0}\right) \subseteq \mathcal{X}$ as the linear span of all the states of $\Sigma$ which are reachable from an initial state in $\mathcal{X}_{0}$ if the continuous-valued inputs and the switching times are arbitrary, but the sequence of discrete modes is precisely $q_{1} q_{2} \ldots q_{k}$, i.e.

$$
W_{q_{1} q_{2} \ldots q_{k}}\left(\mathcal{X}_{0}\right)=\operatorname{Span}\left\{x_{\Sigma}\left(x_{0}, u,\left(q_{1}, t_{1}\right)\left(q_{2}, t_{2}\right) \ldots\left(q_{k}, t_{k}\right)\right) \mid u \in P C(T, \mathcal{U}), t_{1}, t_{2}, \ldots, t_{k} \in T, x_{0} \in \mathcal{X}_{0}\right\}
$$

Notice that $x_{\Sigma}\left(x_{0}, u,\left(q_{1}, t_{1}\right)\left(q_{2}, t_{2}\right) \ldots\left(q_{k}, t_{k}\right)\right)=x_{\Sigma}\left(x_{\Sigma}\left(x_{0}, u, s\right), \operatorname{Shift}_{T_{s}}(u),\left(q_{k}, t_{k}\right)\right)$, where $T_{s}=\sum_{i=1}^{k-1} t_{i}$, and $s=\left(q_{1}, t_{1}\right) \ldots\left(q_{k-1}, t_{k-1}\right)$. Using the fact that in the discrete mode $q_{k}$ the system $\Sigma$ behaves like a bilinear system and using the results from $[1,2]$ one gets that for each fixed $s=\left(q_{1}, t_{1}\right) \ldots\left(q_{k-1}, t_{k-1}\right) \in(Q \times T)^{*}$ and $u \in P C\left(\left[0, \sum_{1}^{k-1} t_{j}\right], \mathcal{U}\right)$ it holds that $W_{q_{k}}\left(\left\{x_{\Sigma}\left(x_{0}, u, s\right)\right\}\right)=\operatorname{Span}\left\{B_{q_{k}, w} x_{\Sigma}\left(x_{0}, u, s\right) \mid w \in \mathrm{Z}_{m}^{*}\right\}$. That is,

$$
W_{q_{1}, \ldots, q_{k}}\left(\mathcal{X}_{0}\right)=\operatorname{Span}\left\{B_{q_{k}, w} x \mid x \in W_{q_{1}, \ldots, q_{k-1}}\left(\mathcal{X}_{0}\right), w \in \mathrm{Z}_{m}^{*}\right\}
$$

Taking into account that by $[1] W_{q}\left(\mathcal{X}_{0}\right)=\operatorname{Span}\left\{B_{q, w} x_{0} \mid x_{0} \in \mathcal{X}_{0}\right\}$ and $\operatorname{Span}\left\{x \mid x \in \operatorname{Reach}\left(\Sigma, \mathcal{X}_{0}\right)\right\}=\operatorname{Span}\{x \mid$ $x \in \mathcal{X}_{0}$ or $x \in W_{q_{1}, \ldots, q_{k}}\left(\mathcal{X}_{0}\right)$, for some $\left.q_{1}, \ldots, q_{k} \in Q, k>0\right\}$, the statement of Part (i) of the theorem follows.

Part (ii). It is easy to deduce from $(2.11)$ of Theorem 2.1 that $y_{\Sigma}(x, .$.$) is linear in x$, hence $y_{\Sigma}\left(x_{1}, . ..\right)=$ $y_{\Sigma}\left(x_{2}, . ..\right)$ is equivalent to $y_{\Sigma}\left(x_{1}-x_{2}, . ..\right)=0$. Hence it is enough to show that

$$
x \in O_{\Sigma} \text { if and only if } y_{\Sigma}(x, ., .)=0 .
$$

To this end, let $\mu_{x}:\left\{y_{\Sigma}(x, .,).\right\} \ni y_{\Sigma}(x, .,.) \mapsto x \in \mathcal{X}$. It is easy to see that $\left(\Sigma, \mu_{x}\right)$ is a realization of $\left\{y_{\Sigma}(x, . .).\right\}$. But then by Theorem 2.5, $y_{\Sigma}(x, . .$.$) has a generalized Fliess-series expansion, i.e. y_{\Sigma}(x, .,)=.F_{c_{x}}$, where the GCGS $c_{x}: \widetilde{\Gamma}^{+} \rightarrow \mathbb{R}^{p}$ is defined as $c_{x}\left(\left(q_{1}, w_{1}\right) \ldots\left(q_{k}, w_{k}\right)\right)=C_{q_{k}} B_{q_{k}, w_{k}} \ldots B_{q_{1}, w_{1}} x$ for all $q_{1}, \ldots$, $q_{k} \in Q, w_{1}, \ldots, w_{k} \in \mathrm{Z}_{m}^{*}, k>0$. By Lemma A.2, $F_{c_{x}}=y_{\Sigma}(x, .,)=$.0 if and only if $c_{x}(s)=0$ for all $s \in \widetilde{\Gamma}^{+}$. But the latter is equivalent to $x \in O_{\Sigma}$. Note that one can also give a proof without using the notion of GCGS and Theorem 2.5. However, that proof is much longer than the one above.

\section{A.3. Proof of technical results from Sections 3.1 and 3.2}

Proof of Lemma 3.2. Recall from Definition 2.4 the definition of the relation $R \subseteq \widetilde{\Gamma}^{*} \times \widetilde{\Gamma}^{*}$. First we prove that if $(l, v) \in R$, then $\phi(l)=\phi(v)$. Indeed, $(l, v) \in R$ implies that there exists $h, s \in \widetilde{\Gamma}^{*}$, such that either (1) $l=h\left(q, w_{1}\right)\left(q, w_{2}\right) s$ and $v=h\left(q, w_{1} w_{2}\right) s$, or (2) $l=h\left(q_{1}, \epsilon\right)\left(q_{2}, w\right) s$ and $v=h\left(q_{2}\right.$, w)s. Notice that $\phi\left(\left(q, w_{1}\right)\left(q, w_{2}\right)\right)=\phi\left(\left(q, w_{1}\right)\right) \phi\left(\left(q, w_{2}\right)\right)=\phi\left(\left(q, w_{1} w_{2}\right)\right)$, and $\phi\left(\left(q_{1}, \epsilon\right)\left(q_{2}, w\right)\right)=\phi\left(\left(q_{1}, \epsilon\right)\right) \phi\left(\left(q_{2}, w\right)\right)=\phi\left(\left(q_{2}, w\right)\right)$. Hence, if (1) holds, then $\phi(l)=\phi(h) \phi\left(\left(q, w_{1}\right)\left(q, w_{2}\right)\right) \phi(s)=\phi(h) \phi\left(\left(q, w_{1} w_{2}\right)\right) \phi(s)=\phi(v)$. If (2) holds, then $\phi(l)=\phi(h) \phi\left(\left(q_{1}, \epsilon\right)\left(q_{2}, w\right)\right) \phi(s)=\phi(h) \phi\left(\left(q_{2}, w\right)\right) \phi(s)=\phi(v)$. Hence, in both cases $\phi(l)=\phi(v)$. Finally, we will prove the general case. Assume that $(l, v) \in R^{*}$. Then either $l=v$, and hence $\phi(l)=\phi(v)$, or there exists $s_{0}, \ldots, s_{k} \in \widetilde{\Gamma}^{*}, k \geq 0$ such that $s_{0}=l$ and $s_{k}=v$ and for all $i=0, \ldots, k-1$, either $\left(s_{i}, s_{i+1}\right) \in R$ or $\left(s_{i+1}, s_{i}\right) \in R$. But by the first part of the proof, $\left(s_{i}, s_{i+1}\right) \in R$ or $\left(s_{i+1}, s_{i}\right) \in R$ both imply $\phi\left(s_{i}\right)=\phi\left(s_{i+1}\right)$, hence $\phi(l)=\phi(v)$.

Proof of Lemma 3.3. We prove it by induction on the length of $v$. If $v=\epsilon$, then the statement of the lemma is trivially true. Assume that the statement of the lemma is true for all words $s \in \widetilde{\Gamma}^{*}$ of length at most $k-1$. Let $v=s\left(q_{k}, w_{k}\right)$ where $s$ is a word of length $k-1$. Assume that $w_{k}=j_{1} j_{2} \ldots j_{l}$ for some $j_{1}, j_{2}, \ldots, j_{l} \in \mathrm{Z}_{m}$ and $l \geq 0$. If $l=0$, i.e. $w_{k}=\epsilon$, then $\phi(v)=\phi(s)$. Notice that $v(q, \epsilon)=s\left(q_{k}, \epsilon\right)(q, \epsilon)$, hence $(v(q, \epsilon), s(q, \epsilon)) \in R \subseteq R^{*}$ by Part 2 of Definition 2.4. In addition, by induction hypothesis we get that $(s(q, \epsilon), \phi(s)(q, \epsilon)) \in R^{*}$. Hence, if $l=0$, then by transitivity of $R^{*}$ and $\phi(s)=\phi(v)$ we get that $(v(q, \epsilon), \phi(v)(q, \epsilon)) \in R^{*}$. Assume now that $l>0$. We will show that $(v, \phi(v)) \in R^{*}$, from which the statement of the lemma follows easily. For each $i=0, \ldots, l$, let 
$v_{i}=\phi(s)\left(q_{k}, j_{1}\right)\left(q_{k}, j_{2}\right) \ldots\left(q_{k}, j_{i}\right)\left(q_{k}, j_{i+1} \ldots j_{l}\right)$. It follows that $v_{0}=\phi(s)\left(q_{k}, w_{k}\right)$ and $v_{l}=\phi(v)$. From Part 2, Definition 2.4, $\left(\phi(s)\left(q_{k}, w_{k}\right), \phi(s)\left(q_{k}, \epsilon\right)\left(q_{k}, w_{k}\right)\right) \in R^{*}$ and $\left(s\left(q_{k}, w_{k}\right), s\left(q_{k}, \epsilon\right)\left(q_{k}, w_{k}\right)\right) \in R^{*}$. By induction hypothesis $\left(s\left(q_{k}, \epsilon\right), \phi(s)\left(q_{k}, \epsilon\right)\right) \in R^{*}$, hence by transitivity of $R^{*}$, we get $\left(s\left(q_{k}, w_{k}\right), \phi(v)\left(q_{k}, w_{k}\right)\right)=\left(v, v_{0}\right) \in R^{*}$. For all $i=0, \ldots, l-1,\left(v_{i+1}, v_{i}\right) \in R \subseteq R^{*}$, since $v_{i}=\left(\phi(s)\left(q_{k}, j_{1}\right) \ldots\left(q_{k}, j_{i}\right)\right)\left(q_{k}, j_{i+1} j_{i+2} \ldots j_{l}\right)$ and $v_{i+1}=$ $\left(\phi(s)\left(q_{k}, j_{1}\right) \ldots\left(q_{k}, j_{i}\right)\right)\left(q_{k}, j_{i+1}\right)\left(q_{k}, j_{i+2} \ldots j_{l}\right)$. Combining $\left(v, v_{0}\right) \in R^{*}, v_{l}=\phi(v)$ and $\left(v_{i+1}, v_{i}\right) \in R^{*}$, $i=0, \ldots, l-1$ and using symmetry and transitivity of $R^{*}$ we get that $(v, \phi(v)) \in R^{*}$.

Proof of Lemma 3.10. First, we will show that $\widetilde{c}$ is well-defined map. It is clear that for any $s \in \widetilde{J L}$ there exists a $w \in J L$ such that $(s, w) \in R^{*}$, hence the value of $\widetilde{c}$ for any $s \in \widetilde{J L}$ is defined. Next, we will show that $\widetilde{c}(s)$ is defined uniquely. Indeed, if $(s, w),(s, v) \in R^{*}, w, v \in J L$, then $c(w)=c(v)=\widetilde{c}(s)$, since $c$ was assumed to be a generating convergent series and hence it satisfies Definition 2.5, Condition 1. Next, we will show that $\widetilde{c}$ satisfies Conditions (1)-(2) of Definition 2.5. If $(s, x) \in R^{*}$, then $\widetilde{c}(s)=\widetilde{c}(x)$. Moreover, if $(s, w) \in R^{*}$ and $s=\left(z_{1}, x_{1}\right) \ldots\left(z_{l}, x_{l}\right)$ and $w=\left(q_{1}, v_{1}\right) \ldots\left(q_{k}, v_{k}\right)$, then from the definition of $R$ it follows that $\sum_{1}^{k}\left|v_{i}\right|=\sum_{1}^{l}\left|x_{i}\right|$, that is, $\|\widetilde{c}(s)\|=\|c(w)\| \leq K M^{\sum_{1}^{k}\left|v_{i}\right|}=K M^{\sum_{1}^{l}\left|x_{l}\right|}$. That is, $\widetilde{c}: \widetilde{J L} \rightarrow \mathcal{Y}$ is indeed a GCGS.

Proof of Lemma 3.11. Consider $s \in \Gamma^{*}$. If there exists $w(q, v) \in J L$ such that $(w(q, v), s(q, \epsilon)) \in R^{*}$, then from the definition of $\widetilde{J L}$ it follows that $s(q, \epsilon) \in \widetilde{J L}$, which by the definition of $L_{q}$ implies that $s \in L_{q}$.

Conversely, assume that $s \in L_{q}$. Then $s(q, \epsilon) \in \widetilde{J L}$ and hence there exists a word $\hat{w} \in J L$ such that $(\hat{w}, s(q, \epsilon)) \in R^{*}$. We will argue that $\hat{w}$ can be written as $\hat{w}=w(q, v)$. Notice that $(\hat{w}, s(q, \epsilon)) \in R^{*}$ implies that there exist words $z_{0}=\hat{w}, z_{k}=s(q, \epsilon)$ and $z_{i} \in \widetilde{\Gamma}^{*}, i=1, \ldots, k-1$ such that for each $i=0, \ldots, k-1$, $\left(z_{i}, z_{i+1}\right) \in R$ or $\left(z_{i+1}, z_{i}\right) \in R$ or $z_{i}=z_{i+1}$. In other words, either $z_{i+1}=z_{i}$, or $z_{i+1}$ can be obtained from $z_{i}$ (or vice versa) using one of the two operations described in Definition 2.4. But none of the operations in Definition 2.4 can erase the last discrete state of $z_{i}$ for $i=0, \ldots, k-1$. That is, if $z_{i}=\hat{z}_{i}\left(q_{i}, r_{i}\right)$ for some $q_{i} \in Q$, then necessarily $z_{i+1}=\hat{z}_{i+1}\left(q_{i}, r_{i+1}\right)$. Hence, we get that the last discrete state of $\hat{w}$ and of $s(q, \epsilon)$ coincide, i.e. $\hat{w}=w(q, v)$ for some $w \in \widetilde{\Gamma}^{*}$ and $v \in \mathrm{Z}_{m}^{*}$.

Finally, we present the proof of Lemma 3.12. To this end, we need the following corollary of Lemma 3.11.

Corollary A.1. For each $s \in \Gamma^{*}, s$ belongs to $L_{q}$ if and only if there exists $w(q, v) \in J L$ such that $\phi(w(q, v))=s$.

Proof of Corollary A.1. If $s \in L_{q}$ then there exists $w(q, v) \in J L$ such that $(w(q, v), s(q, \epsilon)) \in R^{*}$ by Lemma 3.11. Then from Lemma 3.2 it follows that $\phi(w(q, v))=\phi(s(q, \epsilon))=\phi(s)$. Conversely, assume that $\phi(w(q, v))=s$ for some $w(q, v) \in J L$. We show that $s \in L_{q}$. From Lemma 3.3 it follows that $(w(q, v)(q, \epsilon), \phi(w(q, v))(q, \epsilon)) \in R^{*}$. Hence, $(w(q, v)(q, \epsilon), s(q, \epsilon)) \in R^{*}$. Notice that $(w(q, v)(q, \epsilon), w(q, v)) \in R$. Using transitivity of $R^{*}$ we get $(w(q, v), s(q, \epsilon)) \in R^{*}$, which by Lemma 3.11 implies that $s \in L_{q}$.

Proof of Lemma 3.12. Define $p r_{Q}: \Gamma^{*} \rightarrow Q^{*}$ by $\operatorname{pr}_{Q}\left(\left(q_{1}, j_{1}\right) \ldots\left(q_{k}, j_{k}\right)\right)=q_{1} \ldots q_{k}$ for each $q_{1}, \ldots, q_{k} \in Q$ and $j_{1}, \ldots, j_{k} \in \mathrm{Z}_{m}$ and $k \geq 0$. Recall from [6], equations (3.20) and (5.11), the definition of the sets $F_{q}(w)$ and $\widetilde{L}_{q}$. Recall from [6], Lemma 5.8, that if $L$ is regular, then $\widetilde{L}_{q}$ is regular. We shall prove that $L_{q}=\operatorname{pr}_{Q}^{-1}\left(\widetilde{L}_{q}\right)$. From this equality it follows that if $\widetilde{L}_{q}$ is regular, then $L_{q}$ is regular. Hence, if $L$ is regular, then $L_{q}$ is regular.

We now proceed with the proof of the equality $L_{q}=p r_{Q}^{-1}\left(\widetilde{L}_{q}\right)$. First, we show that $L_{q} \subseteq p r_{Q}^{-1}\left(\widetilde{L}_{q}\right)$. If $s=\left(q_{1}, j_{1}\right) \ldots\left(q_{r}, j_{r}\right) \in L_{q}$, then by Corollary A.1 there exists an $w \in \widetilde{\Gamma}^{*}$ and $(q, v) \in \widetilde{\Gamma}$ such that $w(q, v) \in$ $J L$ and $\phi(w(q, v))=s$. Assume that $w$ is of the form $w=\left(z_{1}, m_{1}\right) \ldots\left(z_{k}, m_{k}\right)$, where $z_{1}, \ldots, z_{k} \in Q$ and $m_{1}, \ldots, m_{k} \in \mathbf{Z}_{m}^{*}$. Since $w(q, v) \in J L$, we get that $z_{1} z_{2} \ldots z_{k} q \in L$. Let $l$ be the smallest index $j$ such that the word $m_{j}$ is non-empty. From the fact $\phi(w(q, v))=s$ it follows that the following relationship holds between $z_{l}, \ldots, z_{k}$ and $q_{1}, \ldots, q_{r}$; the first $\left|m_{l}\right|$ letters of $q_{1} q_{2} \ldots q_{r}$ coincide with $z_{l}$, for each $i=l, \ldots, k-1$, the letters $q_{\left(\sum_{j=1}^{i}\left|m_{j}\right|\right)+1}, q_{\left(\sum_{j=1}^{i}\left|m_{j}\right|\right)+2}, \ldots, q_{\sum_{j=1}^{i+1}\left|m_{j}\right|}$ equal $z_{i+1}$, and finally the last $|v|$ letters $q_{r-|v|+1}, \ldots, q_{r}$ are equal to $q$. In addition, $|v|+\sum_{i=1}^{k}\left|m_{i}\right|=r$. That is, we get that $q_{1} \ldots q_{r} q=z_{l}^{\left|m_{l}\right|} \ldots z_{k}^{\left|m_{k}\right|} q^{|v|} q$. Define the words $s=z_{1} \ldots z_{l-1}$ and $x=z_{l} \ldots z_{k} q$. Then $s x=z_{1} \ldots z_{k} q \in L$, i.e. $\left(s,\left(\left(\left|m_{1}\right|, \ldots,\left|m_{k}\right|,|v|\right), x\right)\right) \in F_{q}\left(q_{1} q_{2} \ldots q_{r}\right)$. Hence, $q_{1} q_{2} \ldots q_{r}=\operatorname{pr}_{Q}\left(\left(q_{1}, j_{1}\right)\left(q_{2}, j_{2}\right) \ldots\left(q_{r}, j_{r}\right)\right) \in \widetilde{L}_{q}$. That is, $L_{q} \subseteq p r_{Q}^{-1}\left(\widetilde{L}_{q}\right)$. 
Conversely, we show that $\operatorname{pr}_{Q}^{-1}\left(\widetilde{L}_{q}\right) \subseteq L_{q}$. To this end, let $w \in \widetilde{L}_{q}$ and let $(u,(\alpha, h)) \in F_{q}(w)$. Assume that $u$ and $h$ are of the form $u=q_{1} q_{2} \ldots q_{|u|}$ and $h=z_{1} z_{2} \ldots z_{k}$, where $q_{1}, q_{2}, \ldots, q_{|u|} \in Q$, and $z_{1}, z_{2}, \ldots, z_{k} \in Q$ are the letters of $u$ and $h$. From the definition of $F_{q}(w)$ it follows that $w=z_{1}^{\alpha_{1}} z_{2}^{\alpha_{2}} \ldots z_{k}^{\alpha_{k}}$ where $\alpha=\left(\alpha_{1}, \alpha_{2}, \ldots, \alpha_{k}\right) \in$ $\mathbb{N}^{k}$, and $z_{k}=q$. Notice that for any $v \in \Gamma^{*}, v \in p r_{Q}^{-1}(w)$ if and only if $v=v_{1} v_{2} \ldots v_{k}$, where for each $i=1, \ldots, k, v_{i}=\left(z_{i}, j_{1, i}\right)\left(z_{i}, j_{2, i}\right) \ldots\left(z_{i}, j_{\alpha_{i}, i}\right) \in \Gamma^{*}$, for some $j_{1, i}, j_{2, i}, \ldots, j_{\alpha_{i}, i} \in \mathrm{Z}_{m}$. Define the words $\underline{j_{i}}=j_{1, i} j_{2, i} \ldots j_{\alpha_{i}, i} \in \mathbf{Z}_{m}^{*}$. Consider the word $s=\left(q_{1}, \epsilon\right)\left(q_{2}, \epsilon\right) \ldots\left(q_{|u|}, \epsilon\right)\left(z_{1}, j_{1}\right)\left(z_{2}, j_{2}\right) \ldots\left(z_{k}, j_{k}\right)$ in $\widetilde{\Gamma}^{*}$. Since $\overline{u v}=q_{1} q_{2} \ldots q_{|u|} z_{1} z_{2} \ldots z_{k} \in L$, it follows that $s \in J L$. In addition, notice that $\phi(s) \equiv v$ and recall that $z_{k}=q$. By Corollary A.1 the latter implies that $v \in L_{q}$. That is, $\operatorname{pr}_{Q}^{-1}\left(\widetilde{L}_{q}\right) \subseteq L_{q}$, and consequently $L_{q}=\operatorname{pr}_{Q}^{-1}\left(\widetilde{L}_{q}\right)$.

\section{REFERENCES}

[1] P. D'Alessandro, A. Isidori and A. Ruberti, Realization and structure theory of bilinear dynamical systems. SIAM J. Control 12 (1974) 517-535.

[2] A. Isidori, Nonlinear Control Systems. Springer-Verlag (1989).

[3] D. Liberzon, Switching in Systems and Control. Birkhäuser, Boston (2003).

[4] M. Petreczky, Realization theory for bilinear switched systems, in Proceedings of 44th IEEE Conference on Decision and Control (2005). [CD-ROM only.]

[5] M. Petreczky, Realization Theory of Hybrid Systems. Ph.D. Thesis, Vrije Universiteit, Amsterdam (2006). [Available online at: http://www.cwi.nl/ mpetrec.]

[6] M. Petreczky, Realization theory linear and bilinear switched systems: A formal power series approach - Part I: Realization theory of linear switched systems. ESAIM: COCV (2010) DOI: $10.1051 / \mathrm{cocv} / 2010014$.

[7] E.D. Sontag, Realization theory of discrete-time nonlinear systems: Part I - The bounded case. IEEE Trans. Circuits Syst. 26 (1979) 342-359.

[8] Y. Wang and E. Sontag, Algebraic differential equations and rational control systems. SIAM J. Control Optim. 30 (1992) $1126-1149$. 